Aus der Klinik für Kardiologie und Pneumologie

(Prof. Dr. med. Gerd Hasenfuß)

im Zentrum Innere Medizin

der Medizinischen Fakultät der Universität Göttingen

\title{
Einfluss der Gebrechlichkeit auf Morbidität und \\ Mortalität nach kathetergestützter \\ Aortenklappenimplantation (TAVI)
}

\author{
INAUGURAL-DISSERTATION \\ zur Erlangung des Doktorgrades \\ der Medizinischen Fakultät der \\ Georg-August-Universität zu Göttingen
}

vorgelegt von

Bettina Sobisiak geb. Momberger

aus Berlin

Göttingen 2016 
Dekan:

I. Berichterstatter:

II. Berichterstatter:

III. Berichterstatterin:

Tag der mündlichen Prüfung:
Prof. Dr. rer. nat. H. K. Kroemer

Prof. Dr. med. W. Schillinger

Prof. Dr. med. R. Nau

Prof. Dr. hum. biol. Margarete Schön

21.06.2017 
Abkürzungsverzeichnis

\section{Inhaltsverzeichnis}

1. Einleitung 1

1.1 Hintergrund 1

1.2 Aortenklappenstenose 3

1.2.1 Ätiologie und Pathogenese 3

1.2.2 Klassifikation 4

1.2.3 Klinik der degenerativen Aortenklappenstenose 4

1.2.4 Therapie $\quad 5$

$\begin{array}{ll}1.3 \text { Zielsetzung } & 11\end{array}$

2. Material und Methoden 12

$\begin{array}{ll}2.1 \text { Studiendesign } & 12\end{array}$

2.2 Präoperative Untersuchungen 13

2.2.1 Echokardiographie 13

2.2.2 Angiographie 14

2.2.3 Geriatrische Beurteilung 14

2.3 Indikationsstellung und Patientenauswahlverfahren 16

2.3.1 Ausschlusskriterien 17

2.3.2 Auswahl des Zugangsweges 18

2.3.3 Abschätzung des perioperativen Mortalitätsrisikos mit Hilfe chirurgischer Risikoscores $\quad 18$

2.4 Kathetergestützte Aortenklappenimplantation 19

2.4.1 Transkatheter-Aortenklappenprothesen 19

2.4.2 Vorbereitung des Eingriffs 20

2.4.3 Der transfemorale Aortenklappenersatz 21

2.4.4 Der transapikale Aortenklappenersatz 21

2.5 Postoperative Evaluation 22

2.6 Telefonisches Follow-up 22

$\begin{array}{ll}2.7 \text { Statistik } & 24\end{array}$ 
3. Ergebnisse 25

3.1 Patientenbezogene Informationen 25

$\begin{array}{ll}3.1 .1 \text { Altersverteilung } & 25\end{array}$

3.1.2 Geschlechtsverteilung 26

3.2 Basisdemographische Charakteristika 26

$\begin{array}{ll}3.3 \text { Intrahospitaler Verlauf } & 28\end{array}$

3.4 Analyse der Gesamtmortalität im Langzeitverlauf 30

3.5 Der Einfluss der Gebrechlichkeit 34

3.5.1 Basisdemographische und prozedurale Charakteristika 34

3.5.2 Intrahospitaler Verlauf $\quad 34$

3.5.3 Analyse von Morbidität und Mortalität im Langzeitverlauf 37

3.6 NYHA-Status vor und nach TAVI 41

3.7 Subjektive Beurteilung der Lebensqualität 42

4. Diskussion 43

5. Zusammenfassung 50

6. Anhänge 52

7. Literaturverzeichnis 56 


\section{Abkürzungsverzeichnis}

ACC

Al

ATL

AÖF

AS

$\mathrm{BMI}$

COPD

ESC

EF

Euro SCORE

F

GFR

KHK

$\mathrm{KI}$

KÖF

LDL

LVEF

MI

MW

NYHA
American College of Cardiology

Aortenklappeninsuffizienz

Aktivitäten des täglichen Lebens

Aortenklappenöffnungsfläche

Aortenklappenstenose

Body-Mass-Index

chronisch obstruktive Lungenerkrankung

European Society of Cardiology

Ejektionsfraktion

European System for Cardiac Operative Risk Evaluation

French

glomeruläre Filtrationsrate

koronare Herzkrankheit

Konfidenzintervall

Klappenöffnungsfläche

low density lipoprotein

linksventrikuläre Ejektionsfraktion

Mitralklappeninsuffizienz

Mittelwert

New York Heart Association 
PAP sys pulmonalarterieller systolischer Druck

PAVK periphere arterielle Verschlusskrankheit

STS-SCORE Society of Thoracic Surgeons Score

TAVI kathetergestützte Aortenklappenimplantation

TEE transösophageale Echokardiographie

TI Trikuspidalinsuffizienz

TTE transthorakale Echokardiographie

VARC Valve Academic Research Consortium

VHF Vorhofflimmern 


\section{Abbildungsverzeichnis}

Abbildung 1: $\quad$ Schematische Darstellung der Zugangs- 8 möglichkeiten für TAVI

Abbildung 2: $\quad$ Diagramm zum Management der schweren Aortenklappenstenose

$\begin{array}{lll}\text { Abbildung 3: } & \text { Katz-Index-Erhebungsbogen } & 16\end{array}$

Abbildung 4: $\quad$ Alterstruktur des Patientenkollektivs 25

Abbildung 5: $\quad$ Geschlechtsverteilung des Patientenkollektivs 26

Abbildung 6: $\quad$ Vergleich der mittels Risikoscores erhobenen $\quad 35$ Schätzwerte mit der tatsächlichen 30-TagesMortalität der TAVI-Patienten

Abbildung 7: $\quad$ Langzeitmortalität der gebrechlichen vs. nicht-gebrechlichen Patienten

Abbildung 8: $\quad$ Subgruppenanalyse zur Differenzierung der Langzeitmortalität zwischen Patienten mit Katz-Index 0-2 und Katz-Index 3-5

Abbildung 9: $\quad$ Landmark-Analyse Tag 0-30 40

$\begin{array}{lll}\text { Abbildung } 10 \quad \text { Landmark-Analyse ab Tag } 31 & 40\end{array}$

Abbildung 11: $\quad$ NYHA-Status vor TAVI (300 Patienten) 41

Abbildung 12: $\quad$ NYHA-Status nach TAVI (183 Patienten) 41 


\section{Tabellenverzeichnis}

$\begin{array}{lll}\text { Tabelle 1: } & \text { Basisdemographische Charakteristika } & 27\end{array}$

Tabelle 2: $\quad$ Periprozedural aufgetretene Komplikationen 29

Tabelle 3: $\quad$ Todesursachen bei gebrechlichen und nicht-gebrechlichen Patienten

Tabelle 4: $\quad$ Prädiktoren der Langzeitmortalität in

33 uni- und multivariaten Analysen 


\section{Einleitung}

\subsection{Hintergrund}

Bevölkerungsrepräsentativen Erhebungen zufolge erreichen immer mehr Menschen in den Industrienationen ein höheres Lebensalter. Mit dem gestiegenen Durchschnittsalter der Bevölkerung nimmt gleichzeitig die Anzahl von Personen mit altersbedingten degenerativen Erkrankungen zu. Der häufigste degenerativ bedingte Herzklappenfehler in Europa und Nordamerika ist die degenerative Aortenklappenstenose (AS) (lung et al. 2003, Nkomo et al. 2006). Ihre Prävalenz in der Altersgruppe der über 75-Jährigen liegt bei etwa $4.8 \%$ (Nkomo et al. 2006).

Gegenwärtig ist der konventionell-chirurgische Herzklappenersatz das Standardverfahren zur Behandlung hochgradiger Klappenvitien. Im Rahmen des Euro Heart Survey (eines europäischen prospektiven Beobachtungsprogrammes) wurden Daten von 5001 Patienten untersucht, die an insgesamt 92 Zentren aufgrund eines schweren Herzklappenfehlers stationär aufgenommen wurden und einen konventionellen Klappenersatz erhalten sollten (lung et al. 2003). Von den Patienten mit schwerer Aortenklappenstenose wurden in diesem Kollektiv $33 \%$ wegen eines zu hohen Risikos letztlich jedoch nicht operiert. Angeführte Argumente waren vor allem eine reduzierte linksventrikuläre Ejektionsfraktion und ein fortgeschrittenes Lebensalter, aber auch multiple Komorbiditäten (lung et al. 2005).

Die Prognose bei symptomatischer Aortenklappenstenose ist schlecht und wird durch konservativ-medikamentöse Therapie nicht wesentlich verbessert: Die mediane Überlebenszeit liegt bei weniger als zwei Jahren und verkürzt sich bei zusätzlich bestehender manifester Herzinsuffizienz auf weniger als ein Jahr (Horstkotte und Loogen 1988). Bis vor wenigen Jahren gab es deshalb für die Patienten, bei denen aufgrund eines zu hohen operativen Risikos auf eine chirurgische Intervention verzichtet werden musste, als einzige lebensverlängernde Alternative die Ballonvalvuloplastie, deren Ergebnis jedoch bekanntermaßen nicht von langer Dauer ist (Otto et al. 1994). Um dieser Gruppe der als inoperabel geltenden Hochrisikopatienten eine darüber 
hinausgehende Behandlungsmöglichkeit zu eröffnen, wurden im vergangenen Jahrzehnt kathetergestützte Verfahren für den Herzklappenersatz entwickelt (Schaefer und Bertram 2010). Im April 2002 erfolgte erstmalig die perkutane Implantation einer ballonexpandierbaren Aortenklappenbioprothese via Katheter bei einem Menschen (Cribier et al. 2002). Seither wurden weltweit mehr als 200.000 kathetergestützte Aortenklappen-Implantationen (TAVIs) durchgeführt (Holmes und Mack 2015). Da sie ohne Eröffnung des Thorax und den Einsatz einer Herz-Lungen-Maschine durchgeführt wird, stellt die TAVI eine deutlich weniger invasive Therapieoption dar.

Hohes Lebensalter und damit einhergehende Komorbiditäten bedingen sowohl die operativen Risiken als auch die postoperative Lebenserwartung entscheidend mit. Beide Faktoren haben daher wichtige Auswirkungen auf den Entscheidungsprozess. In Übereinstimmung mit aktuellen Leitlinien (Vahanian et al. 2012) ist eine TAVI für Patienten mit hochgradiger symptomatischer Aortenklappenstenose vorgesehen, die entweder inoperabel sind oder ein hohes operatives Risiko haben, deren Lebenserwartung dabei jedoch nicht kürzer als ein Jahr ist.

Trotz dieser Empfehlungen ist die beobachtete mittel- und langfristige Sterblichkeit nach einer TAVI erheblich und lag in der randomisierten PARTNER-Studie nach einem bzw. zwei Jahren bei $24 \%$ bzw. $33 \%$ bei den Hochrisikopatienten (Kodali et al. 2012, Smith et al. 2011) und bei $31 \%$ bzw. $43 \%$ bei den als inoperabel eingestuften Patienten (Leon et al. 2010, Makkar et al. 2012). Die Abschätzung des operativen Risikos einer kathetergestützten Aortenklappenimplantation erfolgt durch klinische Beurteilung des Patienten durch ein interdisziplinäres Herzteam sowie mit Hilfe gängiger chirurgischer Risikoscores. Die zur Verfügung stehenden Scores wie der logistische EuroSCORE I, der EuroSCORE II oder der STS-Score wurden jedoch zur Abschätzung der 30-Tages-Mortalität im Rahmen von Bypassoperationen entwickelt und ermöglichen im Fall von Herzklappenoperationen daher bekanntermaßen keine zufriedenstellende Bewertung des exakten operativen Risikos (und noch weniger des Langzeitüberlebens) eines einzelnen Patienten (Barili et al. 2013, Rosenhek et al. 2012). Darüber hinaus scheinen 
insbesondere in den zumeist deutlich betagten TAVI-Patientenkollektiven noch andere Faktoren, die durch die chirurgischen Scores nicht erfasst werden, eine Rolle zu spielen. Eine renommierte Methode, die Mortalität und einen mutmaßlichen Benefit besser zu prognostizieren und so geeignete Kandidaten für eine TAVI zu erkennen, gibt es bislang noch nicht.

Gebrechlichkeit ist ein häufiger Zustand bei Patienten mit symptomatischer Aortenklappenstenose und wird mit einem schlechteren Outcome nach Herzoperationen assoziiert (Afilalo et al. 2010, Lee et al. 2010). Dennoch geht Gebrechlichkeit nicht als Faktor in die aktuellen Risikoscores ein, und ihr prognostischer Einfluss auf das Outcome nach TAVI wurde bisher unzureichend erforscht.

Das Ziel der vorliegenden Studie war es somit, den Einfluss der Gebrechlichkeit auf die mittel- und langfristige Morbidität und Mortalität nach kathetergestützter Aortenklappenimplantation zu untersuchen.

\subsection{Aortenklappenstenose}

Die Aortenklappenstenose (AS) ist ein Herzklappenfehler, bei dem es zu einer Obstruktion im linksventrikulären Ausflusstrakt kommt. Gemeinsam mit der Mitralklappeninsuffizienz ist sie das häufigste erworbene Klappenvitium beim erwachsenen Menschen.

\subsection{1 Ätiologie und Pathogenese}

Unterschieden werden Aortenklappenstenosen kongenitaler und erworbener Ursache. Während kongenitale Vitien in der Regel im frühen Erwachsenenalter manifest werden, wird die erworbene AS häufig erst im höheren Lebensalter (>60. Lebensjahr) symptomatisch.

Insgesamt ist die erworbene Aortenklappenstenose in den westlichen Ländern hauptsächlich degenerativen Ursprungs. Aufgrund adäquater antibiotischer Therapie ist die Inzidenz der rheumatisch bedingten Klappenfehler in den letzten Jahrzehnten dramatisch zurückgegangen und spielt nur noch eine untergeordnete Rolle. 
Sowohl pathogenetisch als auch hinsichtlich der Risikofaktoren weist die senile Form der Aortenklappenstenose einige Ähnlichkeiten zur Arteriosklerose auf. Die Pathogenese der degenerativen AS ist multifaktoriell: Neben hohen flussbedingten Scherkräften, die mechanisch als Stressor einwirken, sind auch entzündliche Prozesse und kardiovaskuläre Risikofaktoren an ihrer Entstehung und Progredienz beteiligt. Zunächst kommt es nach subendothelialer Akkumulation von LDL zu komplexen inflammatorischen Prozessen, die wiederum zur Transformation der valvulären Myofibroblasten in Osteoblasten führen. Es kommt auf diese Weise zu einem Umbau der extrazellulären Matrix mit Fibrosierung und schließlich zur Kalzifizierung der Klappentaschen mit konsekutiver Stenosierung der linksventrikulären Ausflussbahn (Carità et al. 2016, Otto 2008). Assoziierte Risikofaktoren für das Auftreten und die Progression der sklerokalzifizierenden AS sind wie bei der Arteriosklerose eine Hyper-cholesterinämie, arterielle Hypertension, Diabetes mellitus und Tabakkonsum.

\subsubsection{Klassifikation}

Die Schwere der Aortenklappenstenose wird in drei Grade eingeteilt (leicht, mittel, schwer). Zur Klassifikation des Schweregrades der AS wurden sowohl von der European Society of Cardiology (ESC) als auch vom American College of Cardiology (ACC) Leitlinien herausgegeben (Bonow et al. 1998, Vahanian et al. 2012). Zur Ermittlung des Schweregrades werden die Klappenöffnungsfläche (nach der Kontinuitätsgleichung) in $\mathrm{cm}^{2}$, die Ausstromgeschwindigkeit sowie der mittlere Druckgradient eruiert. Die Quantifizierung dieser Parameter erfolgt mittels Dopplersonographie.

\subsubsection{Klinik der degenerativen Aortenklappenstenose}

Die klinische Manifestation der Aortenklappenstenose hängt entscheidend vom Stenosegrad und damit von der Klappenöffnungsfläche ab. In der Regel bleiben leichte und mittelgradige Stenosen symptomlos. Bei stark verringerter Klappenöffnungsfläche $\left(<1 \mathrm{~cm}^{2}\right)$ kann es zum Auftreten der drei sog. Kardinalsymptome der Aortenklappenstenose kommen: Zunehmende Dyspnoe 
bei Belastung, Angina Pectoris und Synkopen. Diese Symptome können gleichzeitig zu Tage treten; häufig zeigen die Patienten jedoch zunächst auch nur eines der genannten Symptome, z. B. klagen sie über zunehmende Luftnot bei körperlicher Aktivität und nachlassende Leistungsfähigkeit.

Allerdings kann ein Auftreten der typischen Symptomatik lange Zeit vollständig ausbleiben: Da durch die verengte Klappe eine pathologische Druckdifferenz zwischen dem prä- und poststenotischen Abschnitt herrscht, führt die chronische Druckbelastung des linken Ventrikels zu einer konzentrischen Linksherzhypertrophie. Trotz der Stenose kann das Herz somit zunächst höhere linksventrikuläre Drücke aufbauen und noch genügend Volumen auswerfen, so dass selbst Patienten mit hochgradiger AS jahrelang symptomlos bleiben können. Werden die Patienten jedoch nicht rechtzeitig therapiert, kommt es im Spätstadium zur linksventrikulären Dilatation mit niedriger Ejektionsfraktion (EF) und daraus resultierend zur Herzinsuffizienz.

\subsubsection{Therapie}

Entsprechend der Klassifikation der Aortenklappenstenose wird bei den Patienten eine Therapie unter Berücksichtigung der derzeitigen Leitlinien eingeleitet (Vahanian et al. 2012).

\subsubsection{Konservative Therapie}

Die medikamentöse Behandlung eignet sich nicht zur Verhinderung der Progredienz der Aortenklappenstenose und stellt keine kausale Heilmethode dar (Horstkotte und Loogen 1988). Laut den aktuellen Leitlinien wird eine alleinige medikamentöse Therapie nur bei Patienten mit leichter und asymptomatischer Aortenklappenstenose empfohlen, bei denen eine Operationsindikation noch nicht gegeben ist. Auch wenn die Symptomatik bei diesen Patienten durch die Medikamentengabe gebessert wird, sollten sie dennoch in regelmäßigen Abständen kardiologisch kontrolliert werden, um eine Progredienz frühzeitig zu erkennen. Patienten mit bereits symptomatischer Aortenklappenstenose sollten nur dann primär konservativ therapiert werden, wenn sie aufgrund hoher operativer Risiken und drastisch verkürzter 
Lebenserwartungen weder chirurgisch noch kathetergestützt operiert werden können (siehe auch Abb. 2).

\subsubsection{Konventionell-chirurgische Therapie}

Goldstandard für die Therapie der hochgradigen symptomatischen Aortenklappenstenose (und anderer schwerer Klappenvitien) ist nach wie vor die konventionelle Herzchirurgie. Es wird ein möglichst früher Ersatz der nativen Klappe angestrebt, da Studien zeigten, dass bei symptomatisch gewordenen Patienten die Mortalität nach drei Jahren bereits bei > 50 \% liegt (Horstkotte und Loogen 1988). Ein hohes Lebensalter an sich stellt keine Kontraindikation für den offen chirurgischen Herzklappenersatz dar. Selbst Patienten im Alter von über 80 Jahren profitieren nach erfolgreicher Operation von einer höheren Lebensqualität und längeren Lebensdauer (Chukwuemeka et al. 2006). Hochrisikopatienten oder Patienten mit gewissen Begleiterkrankungen werden jedoch, wie einleitend bereits erwähnt, sehr häufig als inoperabel eingestuft. Die klassische Herzchirurgie ist mit einem hohen operativen Trauma assoziiert, da dieser Eingriff immer eine Thorakotomie und den Einsatz der Herz-LungenMaschine sowie eine Kardioplegie erforderlich macht. Die Letalität dieses Eingriffs liegt bei $1-3 \%$ bei Patienten unter 70 Jahren und steigt bei älteren Patienten auf 4-8\% (Vahanian et al. 2012).

\subsubsection{Kathetergestützte Aortenklappenimplantation (TAVI)}

Patienten, die aufgrund eines nicht vertretbaren Mortalitätsrisikos nicht der konventionellen Herzchirurgie zugeführt werden, steht seit einigen Jahren mit der TAVI eine kurative Alternative zur Verfügung. Mittels dieses kathetergestützten Verfahrens wird über unterschiedliche Zugangswege (transfemoral, transapikal, transaortal oder durch die A. subclavia, vgl. Abb. 1) die kalzifizierte Nativklappe mittels Ballonvalvuloplastie aufgedehnt. Anschließend wird eine auf einen Stent aufgezogene biologische Klappenprothese im Aortenklappenring platziert und übernimmt die Funktion der ursprünglichen Klappe, wobei die Taschen der nativen Aortenklappe an die Aortenwand gedrückt werden. Im Frühjahr 2002 erfolgte durch Alain Cribier und 
seine Arbeitsgruppe die erste kathetergeführte Implantation einer Aortenklappe beim Menschen. Der Zugangsweg erfolgte transfemoral und damals noch antegrad-transvenös, d.h. über die Vena femoralis und Punktion des interatrialen Septums (Cribier et al. 2002). Dieses komplizierte und nicht ganz risikolose Verfahren wurde etwas später modifiziert, so dass die transfemorale TAVI über einen retrograd-transarteriellen Zugangsweg verlief. Dieses Verfahren nach Webb erfolgt durch die Einführung des Katheters in die Femoralarterie, wobei die Klappe retrograd über Aorta und Aortenbogen vorgeschoben wird, bis sie im Aortenanulus platziert werden kann (Webb et al. 2006). Im klinischen Alltag bewährt und bis heute im Einsatz sind nun der transfemorale retrograd-transarterielle Aortenklappenersatz und der transapikale antegrad-transarterielle Aortenklappenersatz. Der transapikale Zugangsweg erfordert eine laterale Minithorakotomie, geht also mit einem minimalen operativen Trauma einher.

Seltener wird die Aortenklappe über alternative Zugangswege via A. subclavia oder transaortal ersetzt. Verschiedene Zugangsverfahren bei schwerer Aortenklappenstenose sind in Abbildung 1 illustriert: 
Edwards Sapien-Prothese

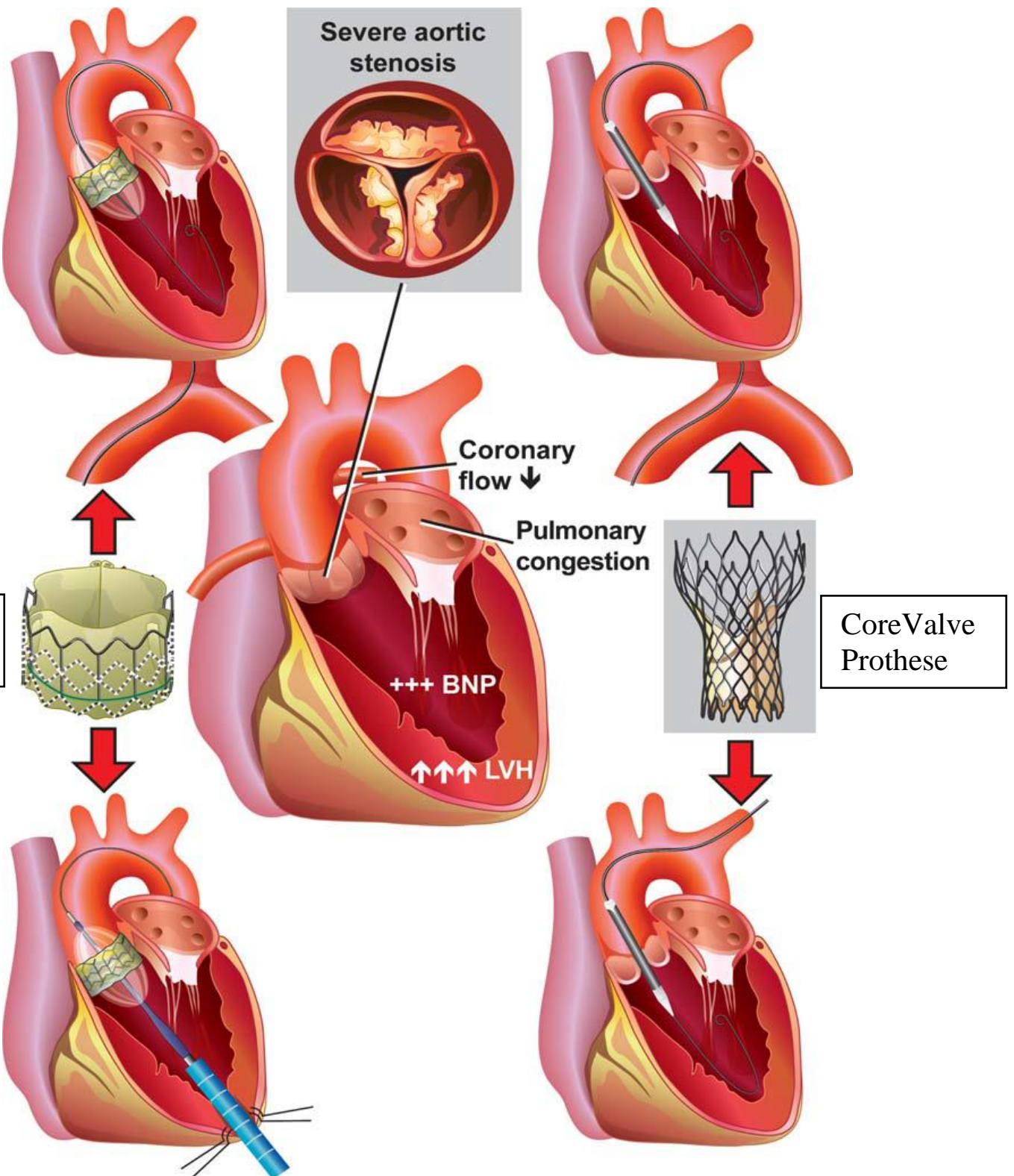

Abb. 1: Schematische Darstellung der Zugangsmöglichkeiten für TAVI (von links oben im Uhrzeigersinn: transfemoral mit Edwards Sapien-Klappe, transfemoral mit CoreValve Klappe, transsubklavikulär mit CoreValve Klappe, transapikal mit Edwards Sapien-Klappe). (Quelle: modifiziert nach Buellesfeld L, Windecker S: Transcatheter aortic valve implantation: the evidence is catching up with reality. Eur Heart J $\underline{32}$, S. 134)

Die Effektivität der kathetergestützten Aortenklappenimplantation wurde in der wegweisenden randomisierten PARTNER-Studie belegt, die zum einen optimale medikamentöse Therapie (inkl. Ballonvalvuloplastie) vs. TAVI bei inoperablen Patienten (Leon et al. 2010, Makkar et al. 2012) sowie zum anderen den konventionellen chirurgischen Aortenklappenersatz vs. TAVI bei 
Hochrisikopatienten verglich (Kodali et al. 2012, Smith et al. 2011). Dabei konnte in der inoperablen Gruppe ein signifikanter Überlebensvorteil der mittels TAVI gegenüber den konservativ behandelten Patienten gezeigt werden (Überlebensrate nach 2 Jahren $57 \%$ vs. $32 \%, P<0.001$ ). Weiterhin wurde für chirurgische Hochrisikopatienten eine Nicht-Unterlegenheit des kathetergestützten Verfahrens gegenüber dem konventionellen Aortenklappenersatz belegt (Überlebensrate nach 2 Jahren $66 \%$ vs. $61 \%$, $P=0,80)$.

\subsubsection{Empfehlungen der aktuellen Leitlinien}

Basierend auf den Ergebnissen der PARTNER-Studie sollen gemäß aktueller ESC Leitlinien (Vahanian et al. 2012) nur Patienten mittels TAVI behandelt werden, die an einer hochgradigen symptomatischen Aortenklappenstenose leiden, für eine konventionelle Operation aber Kontraindikationen oder ein als inakzeptabel eingeschätztes operatives Risiko aufweisen. Weiterhin "sollten geeignete Patienten eine Lebenserwartung von mehr als einem Jahr haben und möglichst mit einer Steigerung ihrer Lebensqualität rechnen können, wobei man ihre Komorbiditäten mit in Betracht ziehen sollte" (Vahanian et al. 2012). Eine gemeinsame Indikationsstellung von Kardiologen und Herzchirurgen ist für eine TAVI unabdingbare Voraussetzung. Nachfolgend eine Darstellung zur besseren Veranschaulichung des Entscheidungsprozesses: 


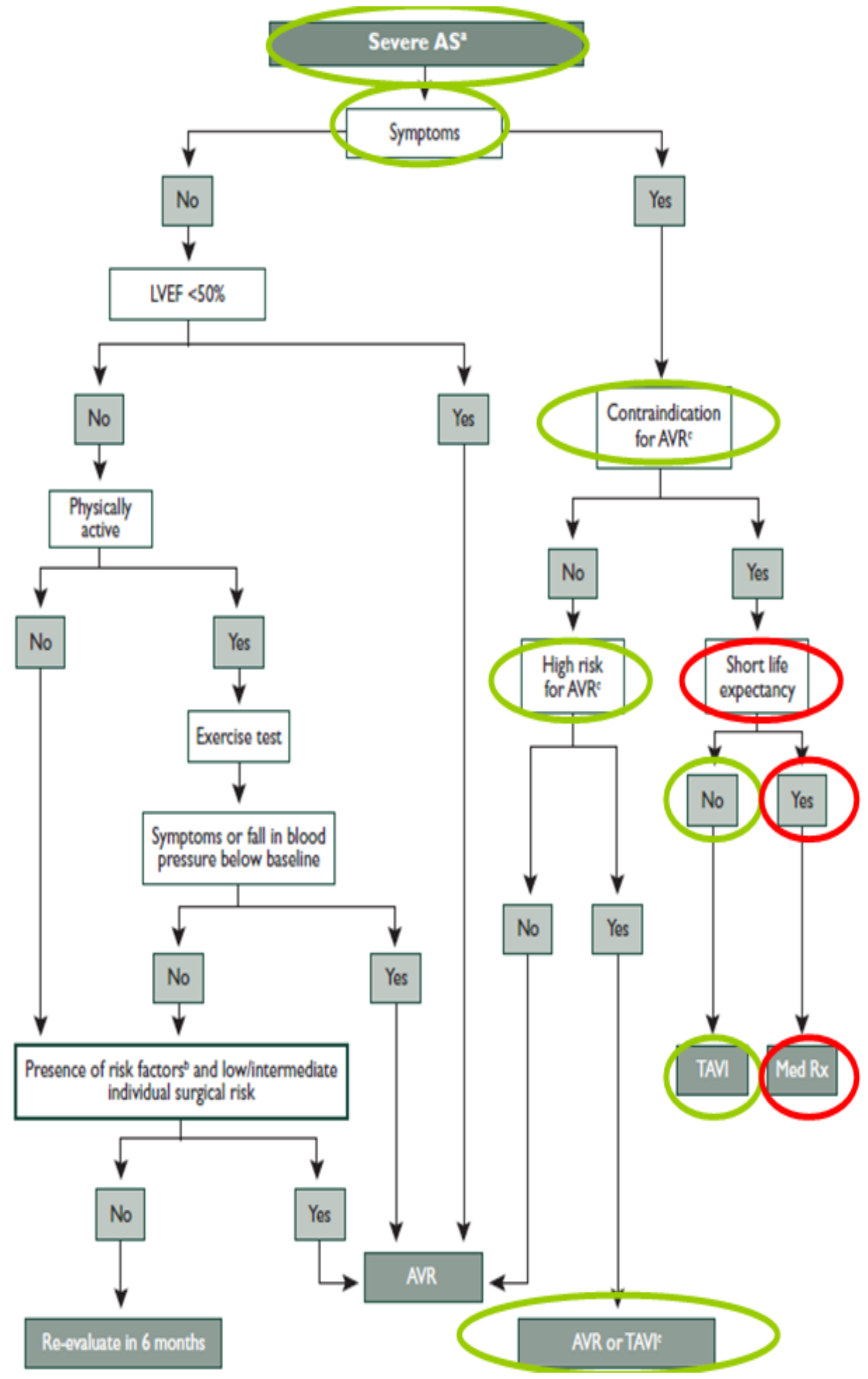

Abb. 2: Diagramm zum Management der schweren Aortenklappenstenose

(Quelle: modifiziert nach Vahanian et al.: Guidelines on the management of valvular heart disease. Eur Heart J $\underline{33}$, S. 2467) 


\subsection{Zielsetzung}

Unser Gesundheitssystem wird mit einer steigenden Anzahl immer älterer, gebrechlicher und multimorbider Patienten mit hochgradiger symptomatischer Aortenklappenstenose, für die keine konventionelle Klappenchirurgie in Frage kommt, konfrontiert. Etabliertes Therapieverfahren dieser speziellen Patientenpopulation ist die kathetergestützte Aortenklappenimplantation.

Das Ziel der vorliegenden Arbeit ist es, mögliche Prädiktoren der Langzeitmortalität nach TAVI zu identifizieren. Hierfür wurden die Daten der ersten 300 konsekutiven Patienten, die am Herzzentrum der Uniklinik Göttingen mittels TAVI behandelt wurden, ausgewertet. Neben der Erfassung der basisdemographischen Charakteristika und der chirurgischen Scores (log. EuroSCORE I und II sowie STS-Score) sollten auch der funktionelle Parameter und der klinische Status der Patienten miteinbezogen werden. Aus diesem Grund erfolgte zusätzlich die Bestimmung von geriatrischen Scores, die die Gebrechlichkeit (frailty) eines Patienten beschreiben. Unter anderem sollte geprüft werden, inwieweit die über diese geriatrischen Scores ermittelte Gebrechlichkeit zur Vorhersage des Langzeitüberlebens nach TAVI geeignet ist. Einbezogen wurden ebenfalls ein prä- und postoperativer Vergleich des NYHA-Stadiums und die subjektive Beurteilung der Patienten bezüglich der Lebensqualität nach dem Eingriff. 


\section{Material und Methoden}

\subsection{Studiendesign}

Im Rahmen der vorliegenden Studie wurden die ersten 300 konsekutiven Patienten untersucht, bei denen im Zeitraum vom 01.08.2008 bis zum 01.03.2012 am Herzzentrum der Universitätsmedizin Göttingen eine kathetergestützte Aortenklappenimplantation erfolgte. Es handelt sich um eine industriell unabhängige monozentrische Beobachtungsstudie. Ein positives Votum der Ethik-Kommission der Universitätsmedizin Göttingen und eine schriftliche Einwilligung der Patienten für den Eingriff selbst sowie für die Erfassung und wissenschaftliche Nutzung der Daten lagen vor.

Die Studienendpunkte wurden vom Valve Academic Research Consortium (VARC) übernommen, welches erstmals 2011 (Leon et al. 2011) und nochmals nach Überarbeitung 2013 (Kappetein et al. 2012) standardisierte Endpunkte für TAVI-Studien vorschlug, um einen Vergleich verschiedener Publikationen zu ermöglichen. Die detailliert im Consensus Report beschriebenen VARC-2 Definitionen (Kappetein et al. 2012) wurden zur Dokumentation periprozeduraler Komplikationen angewandt. Als primärer klinischer Endpunkt wurde (wie durch VARC-2 vorgeschlagen) die Gesamtmortalität gewählt. Die Sterblichkeitsraten wurden für die akute prozedurale Mortalität (innerhalb von 72 Stunden nach TAVI), für die 30-Tages-Mortalität, für die prozedurale Mortalität (definiert als Tod innerhalb von 30 Tagen oder innerhalb des primären Krankenhausaufenthaltes, wenn dieser länger als 30 Tage dauerte) sowie nach sechs Monaten, nach einem Jahr und nach zwei Jahren erfasst. Weiterhin erhoben wir den VARC-definierten kombinierten 30-Tages-Sicherheitsendpunkt bestehend aus den Ereignissen Tod, Schlaganfall jeder Ausprägung, lebensbedrohliche Blutungen, akute Nierenschädigung der Stadien 2 und 3 nach VARC, interventionsbedürftige Obstruktion eines Koronargefäßes, schwerwiegende vaskuläre Komplikationen sowie interventionsbedürftige Funktionsstörung der Klappenprothese. 


\subsection{Präoperative Untersuchungen}

Grundsätzlich erfolgten ein einheitliches Vorgehen zur Ermittlung geeigneter TAVI-Kandidaten und ein standardisiertes Behandlungsverfahren. Alle potentiellen Patienten wurden einem systematischen Prozess der klinischen Evaluation unterzogen.

Neben der gründlichen Anamnese und körperlichen Untersuchung gehörten zu den Basisuntersuchungen auch die Erhebung des NYHA- und CCS-Stadiums anhand des Beschwerdebildes und der Belastungsfähigkeit des Patienten sowie die Abnahme der Laborparameter. Die laborchemische Diagnostik umfasste ein kleines Blutbild, Elektrolyte und Gerinnungsparameter sowie Kreatinin als auch den kardialen Biomarker NT-pro-BNP zur Therapie- und Verlaufskontrolle einer Herzinsuffizienz.

Zur apparativen Diagnostik gehörten neben einem 12-Kanal-EKG und einer Dopplersonographie der Karotiden auch eine transthorakale und transösophageale Echokardiographie (TTE und TEE) sowie eine koronare und aorto-iliofemorale Angiographie bzw. ggf. ergänzend eine Computertomographie. Die mittels Anamnese und o.g. Untersuchungen erhobenen Parameter wurden in einem standardisierten Evaluationsbogen dokumentiert (siehe Anhang 6.1).

\subsubsection{Echokardiographie}

Sowohl für die transösophageale Echokardiographie (TEE) als auch die transthorakale Echokardiographie (TTE) wurden die Echokardiographiegeräte ie33 des Herstellers Philips sowie Vivid E9 des Herstellers GE mit verschiedenen Schallköpfen verwendet. Die echokardiographische Untersuchung und analytische Beurteilung der Echobilder wurden entsprechend den Richtlinien der American Society of Echocardiography von erfahrenen Kardiologen vorgenommen (Zoghbi et al. 2003). Es wurden folgende Parameter erhoben: Die Aortenklappenöffnungsfläche (AÖF) als einer der wichtigsten Parameter zur Klassifikation des Schweregrades der Aortenstenose wurde mittels Kontinuitätsgleichung berechnet. Zur Schweregradeinteilung des Vitiums wurden weiterhin insbesondere der mittlere Gradient sowie die maximale 
Flussgeschwindigkeit über der Aortenklappe (mittels CW-Doppler) bestimmt und in Relation zur linksventrikulären Ejektionsfraktion bewertet. Weitere echokardiographisch bestimmte Größen waren die Weite des Aortenklappenanulus, Graduierung evtl. vorhandener anderer Vitien sowie die linksventrikuläre Ejektionsfraktion. Ferner wurden die linksventrikulären endsystolischen und enddiastolischen Diameter (LVESD und LVEDD) gemessen. Die linksventrikulären Volumina (LVESV und LVEDV) und die Ejektionsfraktion wurden nach der biplanen Methode nach Simpson ermittelt (Lang et al. 2005). Das Vorliegen etwaiger Thromben im linken Vorhofohr wurde mittels TEE ausgeschlossen.

\subsubsection{Angiographie}

Bei jedem Patienten wurde vor geplanter kathetergestützter Aortenklappenimplantation eine Koronarangiographie durchgeführt, um behandlungsbedürttige Stenosen der proximalen Koronarien zu diagnostizieren und ggf. behandeln zu können. Die Angiographie der Becken- und Beingefäße wurde vorgenommen, um deren Größe, Verlauf, Kalzifizierung und Stenosierung beurteilen und so über den günstigsten Implantationsweg entscheiden zu können.

\subsubsection{Geriatrische Beurteilung}

Über die normale klinische Untersuchung hinaus wurden Informationen zur geriatrischen Beurteilung der Patienten wie das Vorliegen einer Gehbehinderung oder einer kognitiven Einschränkung durch geschultes Pflegepersonal dokumentiert. Abhängigkeit bzw. Selbständigkeit in Aktivitäten des täglichen Lebens wurden mit Hilfe des Katz-Index, der Modified Ranking Scale und des Barthel-Index erfasst (siehe auch Anhang 6.2). 


\subsubsection{Katz-Index der Unabhängigkeit bei Ausführung der Aktivitäten des täglichen Lebens (ATLs)}

Der Katz-Index wird auf Katz et. al. (ADL-Index 1963) zurückgeführt und bestimmt, wie unabhängig der Patient bei der Ausführung seiner Aktivitäten des täglichen Lebens ist. Bewertet werden sechs ATLs: Selbstständigkeit beim Essen, beim Transfer vom Bett in den Stuhl, beim Gehen, bei der Benutzung der Toilette, beim Baden und beim Anziehen. Für jede Fähigkeit wird ein Punkt vergeben. Maximal können also sechs Punkte bei völlig unabhängigen Patienten vergeben werden (siehe Abb. 3).

In der vorliegenden Studie wurde jeder Patient mit einem Katz-Index $<6$ als gebrechlich definiert, da bereits eine einzelne Einschränkung auf starke Defizite in der Bewältigung des Alltags hindeutet und in der Regel die Hilfe weiterer Personen oder Pflege erforderlich macht, weil ein Verlust der Selbständigkeit eingetreten ist. 
1. Baden/Waschen

1 Badet/wăscht sich völlig selbständig oder benōtigt Hilfe beim Waschen eines einzelnen Körperteils, wie z.B. Rücken, Intimbereich oder behinderte Extremităt.

0 Benötigt Hilfe beim Waschen von mehr als einem Körperteil, beim Ein- und Aussteigen aus der Badewanne oder der Dusche, oder ist beim Baden auf vollstăndige Hilfe angewiesen.

2. Ankleiden

1 Holt Kleider aus dem Schrank und der Schublade und zieht Wäsche und Kleider selbstăndig an (samt Zuhaken/Zuknöpfen). Benötigt allenfalls Hilfe beim Schuhbinden.

0 Benötigt Hilfe beim selbständigen Ankleiden oder ist auf vollständige Hilfe beim Ankleiden angewiesen.

3. Toilettengang

1 Geht allein auf die Toilette, wischt sich danach selbständig ab und ordnet die Kleidung.

0 Benötigt Hilfe beim Transfer zu der Toilette und beim Abwischen, oder beansprucht Steckbecken oder Toilettenstuhl.

4. Transfer

1 Kommt ohne Hilfe in und aus dem Bett oder einem Sessel. Mechanische Gehhilfen dürfen dabei verwendet werden.

0 Benötigt Hilfe, um vom Bett auf einen Sessel zu gelangen, oder ist auf vollständige Hilfe beim Transfer angewiesen.

5. Kontinenz

1 Besitzt die vollständige Kontrolle über die Harn- und Stuhlentleerung.

0 Leidet an teilweiser oder totaler Stuhl- oder Harninkontinenz.

6. Nahrungsaufnahme

1 Führt das Essen ohne Hilfe vom Teller in den Mund. Die Essensvorbereitung kann von einer anderen Person übernommen werden.

0 Benötigt teilweise oder vollständige Hilfe bei der Nahrungsaufnahme, oder ist auf parenterale Ernährung angewiesen.

\begin{tabular}{|c|c|c|}
\hline Datum & Datum & Datum \\
\hline 0 & 0 & 0 \\
\hline 0 & 0 & 0 \\
\hline 0 & 0 & 0 \\
\hline 0 & 0 & 0 \\
\hline 0 & 0 & 0 \\
\hline 0 & 0 & 0 \\
\hline 0 & 0 & 0 \\
\hline 0 & 0 & 0 \\
\hline 0 & 0 & 0 \\
\hline 0 & 0 & 0 \\
\hline 0 & 0 & 0 \\
\hline 0 & 0 & 0 \\
\hline
\end{tabular}

Total: $(6=$ Hoch [unabhängiger Patient] $0=$ Tief [abhängiger Patient] $)$

$0 / 6$

$0 / 6$

Abb. 3: Katz-Index-Erhebungsbogen

\subsection{Indikationsstellung und Patientenauswahlverfahren}

Die Indikation zur kathetergestützten Aortenklappenimplantation wurde anhand der aktuell gültigen Leitlinien (Vahanian et al. 2012) in der interdisziplinären Herzkonferenz (heart team) gestellt, an welcher u. a. interventionelle Kardiologen und Herzchirurgen der Universitätsmedizin Göttingen teilnahmen. Die im Evaluationsbogen vermerkten Daten wurden im Entscheidungsprozess ebenso berücksichtigt wie der mögliche Benefit und das mittels chirurgischer Scores (EuroSCORE, STS-Score) geschätzte operative Risiko des Patienten. In einem gemeinsamen Positionspapier fordern die Deutsche Gesellschaft für 
Kardiologie (DGK) und die Deutsche Gesellschaft für Thorax-, Herz- und Gefäßchirurgie (DGTHG) eine kritische Indikationsstellung für die kathetergestützte Aortenklappenimplantation (Figulla et al. 2009). Entsprechend dem gegenwärtigen Kenntnisstand erfolgte daher die Patientenauswahl anhand der folgenden Kriterien:

1. Bei den Patienten musste eine schwere Aortenklappenstenose vorliegen. Die Beurteilung des Schweregrades erfolgte via Echokardiographie anhand der ACC/AHA Leitlinien, wobei im Allgemeinen (bei normaler Ejektionsfraktion) die AÖF $<1 \mathrm{~cm}^{2}$, der mittlere Gradient $>40 \mathrm{mmHg}$ und die aortale $\mathrm{V} \max >4 \mathrm{~m} / \mathrm{s}$ betragen musste.

2. Es musste eine symptomatische Aortenklappenstenose mit eingeschränkter Belastbarkeit vorliegen (mind. NYHA Stadium 2).

3. Gemäß den Empfehlungen der ESC sollten nur Patienten mittels TAVI behandelt werden, bei denen ein hohes Operationsrisiko anhand klinischer Evaluation sowie Berücksichtigung der STS- und Euroscores antizipiert werden konnte (Vahanian et al. 2008). Die unteren Grenzwerte für Hochrisikopatienten wurden auf $10 \%$ (STS-Score) bzw. auf $20 \%$ (logistischer Euroscore) festgelegt.

4. Nicht zuletzt wurden auch die nicht von den Risikoscores abgebildeten Faktoren wie hohes Alter, starke Adipositas, Kachexie, Gebrechlichkeit und Immobilität klinisch bewertet. Gleichzeitig wurden anatomische Besonderheiten wie eine Porzellanaorta oder starke Verwachsungen im Thorax aufgrund einer vorangegangenen Bestrahlung berücksichtigt.

\subsubsection{Ausschlusskriterien}

Patienten mit geringer $\mathrm{zu}$ erwartender Operationsletalität sollten einer konventionell chirurgischen Therapie zugeführt werden. Gleiches galt i.d.R. für relativ junge Patienten ( $<75$ LJ) da bislang noch nicht genug Daten zur langjährigen Haltbarkeit dieser minimal-invasiv implantierten biologischen Klappen vorliegen. Kontraindikationen in dieser Studie waren gemäß den ESC 
Leitlinien (Vahanian et al. 2008) bikuspide Aortenklappen, vorhandene apikale linksventrikukäre Thromben sowie Klappendurchmesser von $<18 \mathrm{~mm}$ und $>27 \mathrm{~mm}$.

\subsubsection{Auswahl des Zugangsweges}

Den gängigen Empfehlungen folgend wurde auch die Auswahl des optimalen Zugangsweges in der interdisziplinären Herzkonferenz getroffen (Vahanian et al. 2012). Hierfür wurden Details wie Anatomie der Aortenwurzel und des Aortenbogens, Abstand der Koronararterien von der Klappe, Winkel zwischen Ausflussbahn und Aorta sowie der Durchmesser, Windung und Verkalkungsgrad der peripheren Arterien berücksichtigt. Insbesondere der periphere Gefäßstatus war ein wichtiges Kriterium für die Wahl des adäquaten Zuganges. Die Entscheidung für einen transapikalen Ersatz der Aortenklappe erfolgte bei allen Patienten mit relevanter pAVK, Porzellanaorta, mit massivem Kinking oder Verkalkungen der peripheren Gefäße sowie zu kleinen Durchmessern der Arteria femoralis $(<8 \mathrm{~mm})$. Patienten ohne diese Einschränkungen erhielten in der Regel eine transfemorale Aortenklappenimplantation.

\subsubsection{Abschätzung des perioperativen Mortalitätsrisikos mit Hilfe chirurgischer Risikoscores}

\subsubsection{Logistischer EuroScore}

\section{(European System for Cardiac Operative Risk Evaluation)}

Der logistische EuroScore ist ein mittlerweile etabliertes Werkzeug zur Risikostratifizierung vor Bypassoperationen. Hierzu werden in einem OnlineRechnermodell insgesamt 17 Risikofaktoren erfasst, von denen bekannt ist, dass sie einen prognostischen Einfluss auf die postoperative Mortalität nach operativer Myokardrevaskularisation haben (Roques et al. 2003). Zu diesen Prädiktoren gehören patientenabhängige, eingriffsbedingte und kardial bedingte Risikofaktoren (siehe Anhang 6.3). Für jeden Risikofaktor erfolgt eine 
Punktevergabe, aus der sich dann ein Schätzwert für die 30-Tages-Mortalität in Prozent errechnet (Nashef et al. 1999). Da es sich bei dieser Punktebewertungsform primär um ein Modell für Bypassoperationen handelt, welches schon bei konventionell-chirurgischen Herzklappenoperationen deutlich weniger zuverlässig ist, lassen sich die Ergebnisse nicht eins zu eins auf kathetergestützte Klappenimplantationen übertragen und sind vielmehr im Sinne einer Orientierungshilfe zu verstehen.

\subsubsection{STS-Score (Society of Thoracic Surgeons)}

Auch der STS-Score wurde primär zur Abschätzung des perioperativen Risikos von Bypassoperationen entwickelt und wird online (mittels des OnlineCalculator der Society of Thoracic Surgeons) bestimmt. Neben der Berechnung der 30-Tage-Mortalität ermöglicht er weiterhin die Einschätzung des Risikos spezifischer Komplikationen wie z.B. Schlaganfall oder Nierenversagen und gibt diese ebenfalls in Prozent an. Dementsprechend werden zur Eingabe deutlich mehr Informationen benötigt. Gleichzeitig kann der STS-Score das Risiko verschiedener Eingriffe diskriminieren. So lässt sich auch das Risiko spezieller Klappenoperationen oder einer VAD-Anlage (ventricular assist device) ermitteln. Für die vorliegende Studie wurden für jeden einzelnen Patienten jeweils beide Vorhersagemodelle berechnet.

\subsection{Kathetergestützte Aortenklappenimplantation (TAVI)}

Alle in dieser Studie eingeschlossenen Patienten erhielten einen transfemoralen oder transapikalen kathetergestützten Aortenklappenersatz. In früheren Publikationen wurden diese Eingriffe bereits detailliert beschrieben (Grube et al. 2006, Lichtenstein et al. 2006, Webb et al. 2006).

\subsubsection{Transkatheter-Aortenklappenprothesen}

Beide der zum Studienzeitpunkt in Deutschland zugelassenen Transkatheterklappen wurden eingesetzt. 
28 Patienten erhielten das nur für transfemorale oder transsubclavikuläre Eingriffe zur Verfügung stehende CoreValve ReValving® System der Firma CoreValve (Medtronic, Minneapolis, Minnesota, USA). Dieses Klappensystem besteht aus einem selbstexpandierenden Nitinolrahmen, in welchem drei Aortenklappentaschen aus Schweineperikard befestigt sind. Zum Studienzeitpunkt war es in den Größen 26 und 29 mm verfügbar und konnte mittels einer 18-French-Applikationsschleuse eingebracht werden.

Der Großteil der Patienten (272 Personen) erhielt das sowohl transfemoral als auch transapikal einsetzbare Edwards SAPIEN® (bzw. ab Patient 115 SAPIEN XT) System der Firma Edwards (Irvine, CA, USA). Hierbei handelt es sich um einen ballonexpandierenden Stahlstent mit einer darin befestigten biologischen Klappe aus Rinderperikard. Diese Prothese war zunächst in den Größen 23 mm und $26 \mathrm{~mm}$ erhältlich und wurde bei transfemoralen Prozeduren bis Mai 2010 (Patient 115) mit dem Retroflex 3-Applikationskatheter eingeführt, wofür $22 \mathrm{~F}$ bzw. 24 F-Schleusen benötigt wurden. Nach diesem Zeitpunkt ermöglichte die Einführung des Novaflex-Applikationskatheters den Gebrauch kleinerer Schleusen (18/19 F). Später war auch eine 29 mm-Klappe für die transapikale Implantation erhältlich.

\subsubsection{Vorbereitung des Eingriffs}

Alle TAVIs erforderten die Anwesenheit eines multidisziplinären Teams, bestehend aus jeweils mindestens einem interventionellen Kardiologen und Herzchirurgen, einem Kardio-Anästhesisten, einem Echokardiographiespezialisten sowie spezialisierten Pflegekräften.

Der Eingriff wurde bei transfemoralen Prozeduren im Herzkatheterlabor der Kardiologie der Universitätsmedizin Göttingen und bei transapikalen Prozeduren im OP-Bereich der Herzchirurgie durchgeführt. Die TAVls erfolgten zunächst grundsätzlich unter Intubationsnarkose, später konnten die transfemoralen Eingriffe bei geeigneten Patienten jedoch unter alleiniger Analgosedierung durchgeführt werden. Bei beiden Implantationswegen wurden die Prozeduren mittels TEE-Monitoring begleitet. 


\subsubsection{Der transfemorale Aortenklappenersatz}

Beim transfemoralen Aortenklappenersatz erfolgt der Zugang zum Herzen über die Leistengefäße (Arteria femoralis) (Webb et al. 2006). Zunächst wird die stenosierte Aortenklappe mit Hilfe eines Drahtes passiert, um dann zur Vordehnung eine Ballonvalvuloplastie duchführen zu können. Während der Klappensprengung erfolgt eine schnelle rechtsventrikuläre Stimulation mit einer Herzfrequenz von 180/min oder höher (rapid pacing), um den kardialen Auswurf zu minimieren und damit den Ballon zu stabilisieren. Dann wird die femorale Punktionsstelle schrittweise dilatiert, um letztlich die Einführung der Implantationsschleusen (bei Edwards-Implantationen ab Patient 115 das „NovaFlex System“) zu ermöglichen. Die zwischenzeitlich vorbereitete und nochmal überprüfte Edwards SAPIEN XT Transcatheter Heart Valve wird mittels des Führungskatheters über den Aortenbogen zur Ebene des nativen Aortenklappenannulus vorgeschoben und dort unter Durchleuchtung positioniert. Unter erneutem rapid pacing erfolgt die finale Positionierung durch Ballonexpansion der Klappe, welche die kalzifizierten Taschen der nativen Klappe an die Wand des Aortenannulus drückt. Einer möglicherweise auftretenden relevanten paravalvulären Insuffizienz begegnet man mit Nachdilation. Nach erfolgreicher Klappenimplantation wird das rapid pacing beendet und der NovaFlex Katheter von der Klappe gelöst und zurückgezogen. Der Verschluss der Punktionsstelle erfolgt mittels eines nahtbasierten Verschlusssystems.

Diese Verfahrensweise ist für beide verfügbaren Klappensysteme möglich. Wesentlicher Unterschied ist, dass es sich bei der Edwards Sapien-Klappe um ein ballonexpandierendes System handelt, während das CoreValve Revalving System eine selbstexpandierende Klappe beinhaltet, welche durch Rückzug der äußeren Applikationsschleuse freigesetzt wird (Grube et al. 2006).

\subsubsection{Der transapikale Aortenklappenersatz}

Der Zugang zum Herzen beim transapikalen Aortenklappenersatz erfolgt über die Herzspitze und macht deswegen eine links-anterolaterale Minithorakotomie 
unumgänglich (Lichtenstein et al. 2006). Bei diesem Verfahren erfolgt im Bereich des 5. oder 6. Interkostalraums eine Inzision zur Darstellung der Herzspitze und Eröffnung des Perikards. Zum späteren Verschluss der Punktionsstelle werden dann im Bereich des linksventrikulären Apex filzunterstützte Tabaksbeutelnähte vorgelegt. Unter Durchleuchtung wird die Herzspitze punktiert und ein Führungsdraht sowie eine Applikationsschleuse im linken Ventrikel positioniert. Auch bei diesem Verfahren muss die verkalkte Nativklappe unter rapid pacing mit Hilfe der Ballonvalvuloplastie aufgedehnt werden. Nach Wechsel der Einführungsschleuse wird die Klappenprothese unter fluoroskopischer Kontrolle in der Aortenklappe positioniert und durch Ballonexpansion implantiert. Nach Überprüfung der korrekten Lage und Funktion der Klappe werden der Führungskatheter entfernt und die Tabaksbeutelnähte verschlossen. Danach erfolgen unter vorheriger Einlage von Thoraxdrainagen der Verschluss von Perikard und Hautschnitt.

\subsection{Postoperative Evaluation}

Vor Entlassung der Patienten erfolgte eine Wiederholung der Echokardiographie (in der Regel transthorakal), und alle während des Krankenhausaufenthaltes aufgetretenen Komplikationen und Todesfälle wurden nach den VARC-2 Definitionen dokumentiert (Kappetein et al. 2012). Nach Entlassung erfolgte die Medikation mit $75 \mathrm{mg}$ Clopidogrel für 6 Monate und $100 \mathrm{mg}$ ASS lebenslang. Bei bestehenden Indikationen zur oralen Antikoagulation wie z.B. Vorhofflimmern erhielten die Patienten für 6 Monate ASS zusätzlich zu Marcumar.

\subsection{Telefonisches Follow-up}

Das telefonische Follow-up der 300 Patienten erfolgte mittels eines dafür speziell erstellten Erhebungsbogens (siehe Anhang 6.4). In regelmäßigen Abständen, zuletzt im Februar und März 2013, wurden die Patienten kontaktiert und zu ihrem aktuellen Gesundheitszustand befragt. Hierzu sollten die Patienten u.a. die folgenden Fragen beantworten: 
1. Tritt Luftnot auf?

2. Bei welcher körperlichen Belastung tritt Luftnot auf?

3. Treten Schmerzen in der Brust auf?

4. Kam es seit der Operation zu einem Bewusstseinsverlust?

Einige Fragen sollten auch der Einschätzung des derzeitigen NYHA-Stadiums dienen. Neben der aktuellen klinischen Symptomatik lag das Augenmerk auf weiteren Hospitalisierungen bzw. Todesursachen, falls die Patienten zwischenzeitlich verstorben waren. Anzahl, Gründe und Dauer der Krankenhausaufenthalte wurden dokumentiert und Entlassungsbriefe aus den behandelnden Häusern angefordert. Schwerpunktmäßig interessierte vor allem jede kardiovaskulär bedingte Hospitalisierung. Die Ermittlung der Todesursachen erfolgte durch Kontaktierung der Angehörigen, Hausärzte bzw. behandelnden Krankenhäuser. Auch hier wurden nach Möglichkeit die entsprechenden verfügbaren Unterlagen angefordert.

Außerdem sollte noch die persönliche Beurteilung der Patienten erfragt werden, z. B. ob sie sich noch einmal einer TAVI unterziehen würden. Zur Erfassung der subjektiven Lebensqualität wurde die Entwicklung der Leistungsfähigkeit aus Sicht des Patienten dokumentiert. Dem Patienten standen die Antwortmöglichkeiten "wesentlich verbessert", "leicht verbessert", "unverändert" und "verschlechtert" zur Auswahl. 


\subsection{Statistik}

Die Analyse der Daten und deren statistische Auswertung wurde mit Unterstützung der Abteilung Medizinische Statistik der Universitätsmedizin Göttingen durchgeführt. Die Erfassung und vorläufige Auswertung der erhobenen Parameter erfolgte mit Hilfe von Microsoft Excel 2010. Zur Erstellung der statistischen Datenauswertung und graphischen Aufbereitung der Ergebnisse wurden die Programme graph pad prism version 4.0 und Statistical Computing Software R (version 2.15.1; http://www.r-project.org) verwendet.

Kontinuierliche Variablen wurden in Abhängigkeit des Vorliegens einer Normalverteilung als Mittelwert \pm Standardabweichung oder als Median und Interquartilsabstand (IQR) angegeben. Ein Vergleich dieser Variablen erfolgte über den T-Test oder den Mann Whitney U Test.

Kategoriale Variablen wurden in absoluten Zahlen und Prozentangaben angegeben. Der Vergleich der unterschiedlichen Häufigkeiten dieser Variablen erfolgte über Pearson's Chi-Quadrat-Test. Ein $p<0.05$ wird in dieser Arbeit als statistisch signifikant angenommen, d.h. das Signifikanzniveau wurde auf $5 \%$ festgelegt.

Zur Analyse der Überlebenszeiten wurde die Kaplan-Meier-Methode herangezogen. Diese Methode wurde im Jahre 1958 von den Biostatistikern E. M. Kaplan und P. Meier entwickelt (Kaplan und Meier 1958). Es wurde mittels des Programms R Package Survival untersucht, zu welchem Zeitpunkt das kritische Endereignis (z.B. Tod oder Rehospitalisierung) in Abhängigkeit vom Vorliegen bestimmter Risikomerkmale eingetreten ist. Die graphische Darstellung der Überlebenszeitverteilung erfolgte in Form der Kaplan-MeierKurve, einer umgekehrten Summenhäufigkeitsfunktion mit stufenartiger Anordnung. Die Überlebenszeiten der verschiedenen Gruppen wurden mit dem Logrank -Test verglichen.

Analysen der Überlebenszeit bzw. der ereignisfreien Überlebenszeit wurden für die basisdemographischen Charakteristika, die Risiko-Scores und den KatzIndex durchgeführt. Alle Risikofaktoren, die in der univariaten Analyse eine statistische Signifikanz zeigten, wurden wiederum in einer multivariaten CoxRegressionsanalyse getestet (Cox proportional hazards regression models). 


\section{Ergebnisse}

\subsection{Patientenbezogene Informationen}

\subsubsection{Altersverteilung}

Zur Bestimmung der Altersstruktur wurde als Stichtag das Eingriffsdatum herangezogen. Unsere Patientenkohorte war insgesamt durch ein erhöhtes Alter gekennzeichnet (im Mittel $82.1 \pm 5.3$ Jahre).

10 Patienten waren zum Zeitpunkt des Eingriffs 90 Jahre oder älter.

96 Patienten waren zwischen 85 und 89 Jahre alt.

122 Patienten waren zwischen 80 und 84 Jahre alt.

48 Patienten waren zwischen 75 und 79 Jahre alt.

24 Patienten waren jünger als 75 Jahre.

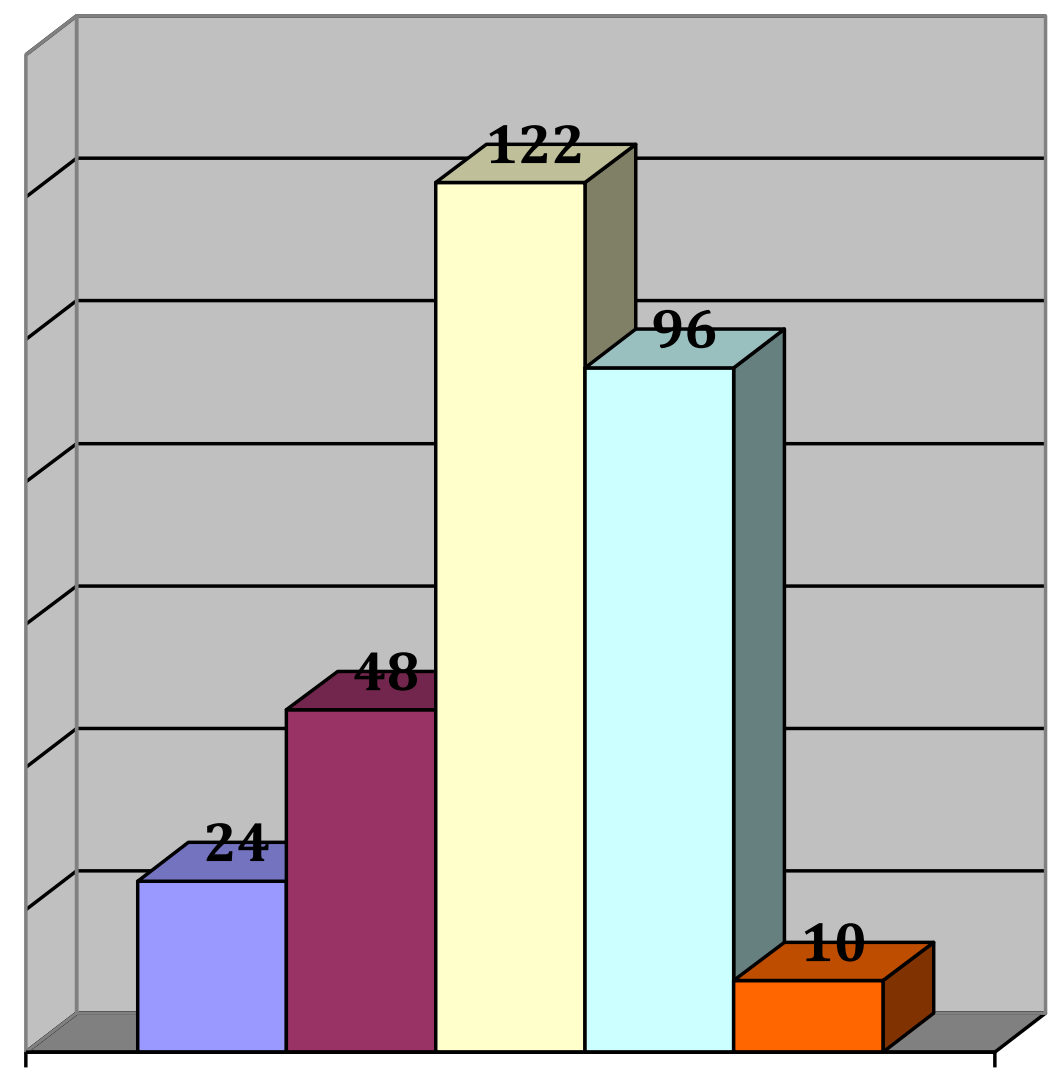

$\square<75 \mathrm{LJ}$

$\square$ 75-79 LJ

$\square$ 80-84 LJ

$\square$ 85-89 LJ

$\square>90$ LJ

Altersstruktur

Abb. 4: Altersstruktur des Patientenkollektivs 


\subsubsection{Geschlechtsverteilung}

Männliches Geschlecht gilt als einer der Risikofaktoren für die degenerative kalzifizierende Aortenklappenstenose. In unserer Kohorte waren ungewöhnlich viele Patienten, nämlich mehr als zwei Drittel der Patienten ( $n=198)$, weiblich.

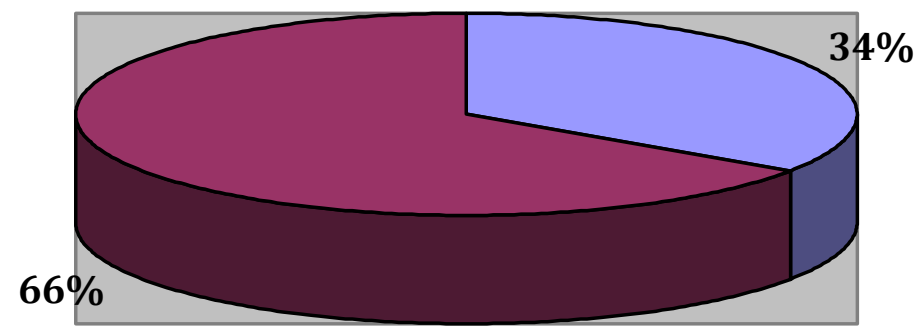

$\square$ Männlich

Weiblich

Abb. 5: Geschlechtsverteilung des Patientenkollektivs

\subsection{Basisdemographische Charakteristika}

Unsere monozentrische TAVI-Kohorte wies neben dem per se hohen Alter auch eine signifikante Anzahl von Komorbiditäten auf, was zu einem messbar erhöhten chirurgischen Risiko führte. Dieses spiegelte sich in den hohen Werten der ermittelten chirurgischen Risikoscores wider (Logistischer EuroSCORE | $26.0 \pm 14.7 \%$, EuroSCORE || $8.5 \pm 7.6 \%$, STS-Score $7.3 \pm$ $5.4 \%$ ). Insgesamt $69 \%$ der Patienten litten unter einer koronaren Herzerkrankung, $59 \%$ unter einer chronischen Niereninsuffizienz (GFR $<30 \mathrm{~mL} / \mathrm{min} / 1.73 \mathrm{~m}^{2}$ in $19 \%$ der Fälle), $34 \%$ unter Diabetes mellitus, $28 \%$ unter COPD, $14 \%$ hatten bereits eine zerebrale Ischämie erlitten, und bei $18 \%$ der Patienten war bereits eine Herzoperation durchgeführt worden (siehe Tabelle 1). Weiterhin wiesen $93 \%$ unserer Patienten ein NYHA Stadium III oder IV auf und waren demnach bereits schwer herzinsuffizient. 144 Personen (48\%) waren in wenigstens einer ATL nach den Katz-Definitionen eingeschränkt und demzufolge als gebrechlich definiert (Katz-Index <6). Demgegenüber waren 156 Individuen (52\%) komplett in ihren ATLs 
unabhängig (Katz-Index = 6). Des Weiteren zeigten 92 Patienten (31\%) Schwächen in der Mobilität und 25 Patienten (8 \%) kognitive Defizite.

\begin{tabular}{|c|c|c|c|c|}
\hline & $\begin{array}{c}\text { Gesamtkollektiv } \\
(n=300)\end{array}$ & $\begin{array}{l}\text { Katz = } 6 \\
(n=156)\end{array}$ & $\begin{array}{l}\text { Katz }<6 \\
(n=144)\end{array}$ & $\mathbf{P}$ \\
\hline Alter, Jahre & $82.1 \pm 5.3$ & $81.2 \pm 5.2$ & $83.1 \pm 5.2$ & $0.0004^{\star}$ \\
\hline Geschlecht, männlich & $102(34 \%)$ & $64(41 \%)$ & $38(26 \%)$ & $0.01^{\star}$ \\
\hline BMI, $\mathrm{kg} / \mathrm{m}^{2}$ & $26.5 \pm 4.7$ & $26.1 \pm 4.2$ & $26.9 \pm 5.1$ & 0.31 \\
\hline KÖF, $/ m^{2}$ & $1.76 \pm 0.27$ & $1.77 \pm 0.27$ & $1.75 \pm 0.28$ & 0.42 \\
\hline \multicolumn{5}{|l|}{ Begleiterkrankungen } \\
\hline$E F<35 \%$ & $35(12 \%)$ & $16(10 \%)$ & $19(13 \%)$ & 0.45 \\
\hline KHK & $206(69 \%)$ & $105(68 \%)$ & $101(70 \%)$ & 0.72 \\
\hline Z.n. PTCA & $83(28 \%)$ & $43(28 \%)$ & $40(28 \%)$ & 0.98 \\
\hline Z.n. Bypass-Operation & $45(15 \%)$ & $29(19 \%)$ & $16(11 \%)$ & 0.06 \\
\hline Z.n. anderer Thorakotomie & $10(3 \%)$ & $7(4.5 \%)$ & $3(2 \%)$ & 0.24 \\
\hline Porzellanaorta & $6(2 \%)$ & $4(3 \%)$ & $2(1 \%)$ & 0.46 \\
\hline Z.n. Bio-AKE & $4(1 \%)$ & $3(2 \%)$ & $1(0.7 \%)$ & 0.35 \\
\hline pAVK & $88(29 \%)$ & $46(30 \%)$ & $42(29 \%)$ & 0.89 \\
\hline Z.n. Apoplex oder TIA & $41(14 \%)$ & $21(14 \%)$ & $20(14 \%)$ & 0.95 \\
\hline COPD & $84(28 \%)$ & $41(27 \%)$ & $43(30 \%)$ & 0.54 \\
\hline Diabetes mellitus & $102(34 \%)$ & $52(34 \%)$ & $50(34 \%)$ & 0.86 \\
\hline GFR $<60 \mathrm{~mL} / \mathrm{min} / 1.73 \mathrm{~m}^{2}$ & $176(59 \%)$ & $86(56 \%)$ & $90(62 \%)$ & 0.25 \\
\hline GFR $<30 \mathrm{~mL} / \mathrm{min} / 1.73 \mathrm{~m}^{2}$ & $56(19 \%)$ & $22(14 \%)$ & $34(23 \%)$ & $0.04^{*}$ \\
\hline \multicolumn{5}{|l|}{ Calculated surgical risk } \\
\hline Logistic EuroSCORE I, \% & $26.0 \pm 14.7$ & $23.6 \pm 12.8$ & $28.8 \pm 16.3$ & $0.005^{\star}$ \\
\hline EuroSCORE II, \% & $8.5 \pm 7.6$ & $7.5 \pm 6.0$ & $9.5 \pm 8.9$ & $0.03^{\star}$ \\
\hline STS Score (Mortalitätsrisiko), & $7.3 \pm 5.4$ & $5.9 \pm 3.2$ & $8.8 \pm 6.7$ & $<0.0001^{*}$ \\
\hline STS Score (Risiko für & $29.8 \pm 11.3$ & $27.1 \pm 8.2$ & $32.8 \pm 13.2$ & $<0.0001^{*}$ \\
\hline \multicolumn{5}{|l|}{ Mortalität oder Morbidität), \% } \\
\hline \multicolumn{5}{|l|}{ Klinische Charakteristika } \\
\hline NYHA-Stadium III & $225(75 \%)$ & $128(82 \%)$ & $97(67 \%)$ & $0.003^{\star}$ \\
\hline NYHA-Stadium IV & $54(18 \%)$ & $15(10 \%)$ & $39(27 \%)$ & $0.0001^{*}$ \\
\hline Periphere Ödeme & $132(44 \%)$ & $63(41 \%)$ & $69(48 \%)$ & 0.23 \\
\hline Ergüsse & $46(15 \%)$ & $24(16 \%)$ & $22(15 \%)$ & 0.94 \\
\hline Feuchte Rasselgeräusche & $96(32 \%)$ & $35(23 \%)$ & $61(42 \%)$ & $0.0003^{\star}$ \\
\hline Synkopen & $68(23 \%)$ & $35(23 \%)$ & 33 (23\%) & 0.97 \\
\hline
\end{tabular}

Tabelle 1: Basisdemographische Charakteristika der TAVI-Kohorte 


\subsection{Intrahospitaler Verlauf}

Die Mortalitätsraten an VARC-definierten Zeitpunkten und die Inzidenzen spezieller Komplikationen sind sowohl für die gesamte Patientenkohorte als auch für gebrechliche und nicht-gebrechliche Patienten in Tabelle 2 aufgelistet. Unmittelbar post-prozedural (d. h. innerhalb von 72 Stunden) verstarben 10 Patienten (3.3\%). Diese Todesfälle traten infolge von kardiogenem Schock $(n=6)$, Aortenwurzelruptur $(n=2)$, Schlaganfall $(n=1)$ und Aspiration $(n=1)$ auf. 33 weitere Patienten starben innerhalb der ersten 30 Tage oder innerhalb des primären stationären Aufenthaltes (wenn dieser länger als 30 Tage dauerte). Ursachen dieser nach VARC-Definition als prozedurale Mortalität bezeichneten Todesfälle waren Schlaganfall $(n=8)$, Pneumonie $(n=6)$, septisch-kardiogener Schock $(n=7)$, kardiogener Schock $(n=2)$, plötzlicher Herztod in der Rehabilitationseinrichtung $(n=2)$, dokumentierte Arrhythmie und erfolglose kardiopulmonale Reanimation ( $n=2)$, hämorrhagischer Schock aufgrund einer Blutung im transapikalen Zugangsweg $(n=2)$, Mesenterialischämie $(n=1)$, Herzbeuteltamponade $(n=1)$, Pleuraempyem $(n=1)$ und hämorrhagischer Schock aufgrund einer oberen gastrointestinalen Blutung $(n=1)$. Die beobachtete 30 -Tage-Mortalität lag bei $11 \%(n=34)$ und wich damit von den geschätzten Werten der chirurgischen Scores teils deutlich ab (logistischer EuroSCORE | $26.0 \pm 14.7 \%$, EuroSCORE || $8.5 \pm 7.6 \%$, STSScore $7.3 \pm 5.4 \%)$.

Den kombinierten VARC-definierten 30-Tage-Sicherheitsendpunkt (bestehend aus 30-Tage-Mortalität, Koronarobstruktion, Schlaganfall, lebensbedrohlichen Blutungen, gravierenden Gefäßkomplikationen, Funktionsstörungen der Klappenprothese und ANV im Stadium 2 oder 3) erreichten 96 Patienten (32\%). Dieser Prozentsatz war relativ gut vom STS-Risikomodell vorhergesagt worden $(29.8 \pm 11.3 \%)$. 


\begin{tabular}{|c|c|c|c|c|}
\hline & $\begin{array}{l}\text { Gesamtkollektiv } \\
\qquad(n=300)\end{array}$ & $\begin{array}{l}\text { Katz = } 6 \\
(n=156)\end{array}$ & $\begin{array}{l}\text { Katz < } 6 \\
(n=144)\end{array}$ & $\mathbf{P}$ \\
\hline Unmittelbare prozedurale Mortalität & $10(3.3 \%)$ & $2(1.3 \%)$ & $8(5.5 \%)$ & $0.04^{\star}$ \\
\hline 30-Tages-Mortalität & $34(11 \%)$ & $9(5.8 \%)$ & $25(17 \%)$ & $0.002^{\star}$ \\
\hline Prozedurale Mortalität & $43(14 \%)$ & $10(6.4 \%)$ & $33(23 \%)$ & $<0.0001^{*}$ \\
\hline Komb. 30d-Sicherheitsendpunkt ${ }^{\star}$ & $96(32 \%)$ & $38(24.4 \%)$ & $58(40.3 \%)$ & $0.003^{*}$ \\
\hline $\begin{array}{l}\text { (nach VARC) } \\
\text { Komb. Endpunkt Morbidität und }\end{array}$ & $94(31 \%)$ & $38(24.4 \%)$ & $56(38.9 \%)$ & $0.007^{\star}$ \\
\hline \multicolumn{5}{|l|}{ Mortalität (nach STS) \# } \\
\hline \multicolumn{5}{|l|}{ Prozedurale Komplikationen } \\
\hline Konversion zu chirurg. AKE & $1(0.3 \%)$ & $1(0.3 \%)$ & 0 & 0.33 \\
\hline Ungeplanter Einsatz der HLM zur & $6(2 \%)$ & $4(2.6 \%)$ & $2(1.4 \%)$ & 0.46 \\
\hline $\begin{array}{l}\text { hämodynamischen Stabilisierung } \\
\text { Ventrikelperforation }\end{array}$ & $2(0.7 \%)$ & $2(1.3 \%)$ & 0 & 0.17 \\
\hline "Valve-in-valve" & $7(2.3 \%)$ & $5(3.2 \%)$ & $2(1.4 \%)$ & 0.29 \\
\hline Koronarobstruktion & $4(1.3 \%)$ & $3(1.9 \%)$ & $1(0.7 \%)$ & 0.35 \\
\hline 2. Klappenintervention (>30d) & $2(0.7 \%)$ & $2(1.3 \%)$ & 0 & 0.17 \\
\hline Myokardinfarkt ${ }^{\star}$ & $4(1.3 \%)$ & $2(1.3 \%)$ & $2(1.4 \%)$ & 0.95 \\
\hline Schlaganfall* & $19(6 \%)$ & $10(6 \%)$ & $9(6 \%)$ & 0.93 \\
\hline TIA $^{*}$ & $2(0.7 \%)$ & $1(0.6 \%)$ & $1(0.7 \%)$ & 0.96 \\
\hline \multicolumn{5}{|l|}{ Blutungskomplikationen } \\
\hline - lebensbedrohlich* & $34(11 \%)$ & $19(12 \%)$ & $15(10 \%)$ & 0.60 \\
\hline - schwer* & $18(6 \%)$ & $6(4 \%)$ & $12(8 \%)$ & 0.11 \\
\hline - leicht $^{\star}$ & $86(29 \%)$ & $36(23 \%)$ & $50(35 \%)$ & $0.03^{\star}$ \\
\hline Perikardtamponade & $8(2.7 \%)$ & $5(3.2 \%)$ & $3(2 \%)$ & 0.53 \\
\hline Bluttransfusionen erhalten, $n$ (\%) & $121(40 \%)$ & $54(35 \%)$ & $67(47 \%)$ & $0.04^{\star}$ \\
\hline $\mathrm{Hb}$ vor TAVI, $g / d L$ & $11.7 \pm 1.7$ & $12.0 \pm 1.7$ & $11.3 \pm 1.6$ & $0.0002^{\star}$ \\
\hline Hb-Abfall nach TAVI, $g / d L$ & $2.9 \pm 1.4$ & $2.8 \pm 1.3$ & $2.9 \pm 1.6$ & 0.90 \\
\hline \multicolumn{5}{|l|}{ Akute Nierenschädigung* } \\
\hline - Stadium 1 & $51(17 \%)$ & $32(21 \%)$ & $19(13 \%)$ & 0.08 \\
\hline - $\quad$ Stadium 2 & $6(2 \%)$ & $1(0.6 \%)$ & $5(3 \%)$ & 0.08 \\
\hline - Stadium 3 & $40(13 \%)$ & $14(9 \%)$ & $26(18 \%)$ & $0.02^{\star}$ \\
\hline Dialyse-Indikation, $n$ (\%) & $40(13 \%)$ & $14(9 \%)$ & $26(18 \%)$ & $0.02^{\star}$ \\
\hline Kreatinin vor TAVI, $m g / d L$ & $1.3 \pm 0.8$ & $1.2 \pm 0.6$ & $1.4 \pm 1.0$ & 0.38 \\
\hline max. Krea bis $7 \mathrm{~d}$ danach, $\mathrm{mg} / \mathrm{dL}$ & $1.7 \pm 1.2$ & $1.5 \pm 1.1$ & $1.9 \pm 1.4$ & $0.03^{*}$ \\
\hline \multicolumn{5}{|l|}{ Zugangskomplikationen* } \\
\hline Schwer $^{*}$ & $26(9 \%)$ & $12(8 \%)$ & $14(10 \%)$ & 0.56 \\
\hline - mit chirurgischem Eingriff & $16(5 \%)$ & $8(5 \%)$ & $8(5 \%)$ & 0.89 \\
\hline - mit perkutanem Eingriff & $11(4 \%)$ & $5(3 \%)$ & $6(4 \%)$ & 0.67 \\
\hline - Aortenannulusruptur & $2(0.7 \%)$ & 0 & $2(1.4 \%)$ & 0.14 \\
\hline Leicht * & $23(8 \%)$ & $12(8 \%)$ & $11(8 \%)$ & 0.96 \\
\hline \multicolumn{5}{|l|}{ Prothesenassoziierte Komplikationen } \\
\hline - $\quad$ Neuer LSB & $14(5 \%)$ & $9(6 \%)$ & $5(3 \%)$ & 0.33 \\
\hline
\end{tabular}




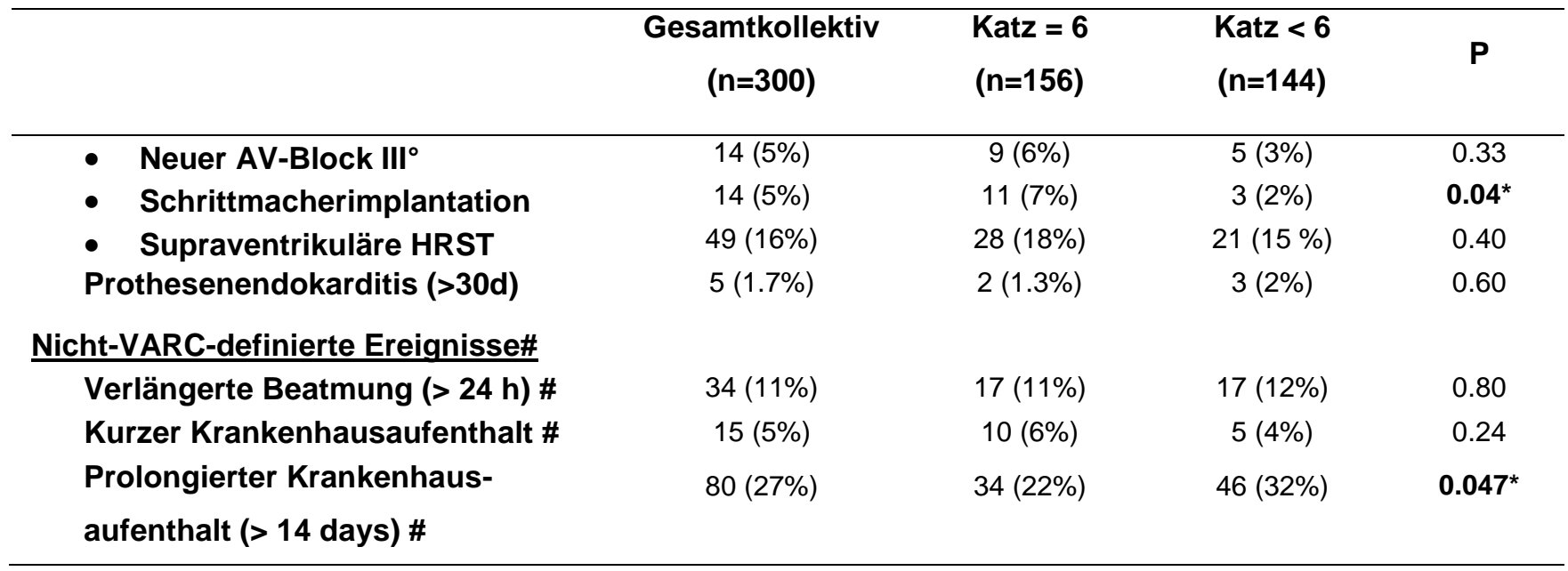

Tabelle 2: Periprozedural aufgetretene Komplikationen

* Definitionen nach den Richtlinien des Valve Academic Research Consortium (VARC-2) .

\# Definitionen nach dem STS Score:

- "Morbidität und operative Mortalität": operative Mortalität, dauerhafter Apoplex, Beatmungsdauer >24 Stunden, schwere Zugangskomplikationen, erneuter Eingriff jeder Ursache

- "Kurzer Krankenhausaufenthalt": Entlassung lebend und innerhalb von 5 Tagen nach OP

- "Prolongierter Krankenhausaufenthalt": Entlassung nicht innerhalb von 14 Tagen nach OP

\subsection{Analyse der Gesamtmortalität im Langzeitverlauf}

Der Überlebensstatus konnte bei allen TAVI-Patienten ermittelt werden. Der mediane Beobachtungszeitraum lag bei 537 Tagen. Das Gesamtüberleben betrug $89 \%$ nach 30 Tagen, $79 \%$ nach 6 Monaten, $72 \%$ nach 12 Monaten und $63 \%$ nach zwei Jahren. Zum Zeitpunkt der letzten Befragung waren 117 Patienten (39\%) verstorben. Von diesen Todesfällen waren $43(14 \%)$ noch zum Zeitpunkt des primären Krankenhausaufenthaltes eingetreten. Die Todesursachen bei den nach der primären Entlassung Verstorbenen $(n=74)$ waren die Folgenden: Herzinsuffizienz $(n=20)$, plötzlicher Herztod $(n=12)$, Malignom $(n=6)$, Pneumonie $(n=6)$, septischer Schock unbekannter Ursache $(n=4)$, Endokarditis der Klappenprothese $(n=2)$, im Verlauf zugezogene Schenkelhalsfraktur $(n=3)$, im Rahmen bzw. nach anderen operativen Eingriffen $(n=2)$, Schlaganfall $(n=1)$, Gefäßkomplikationen $(n=2)$, gastrointestinale Blutungen $(n=2)$, akutes Nierenversagen $(n=1)$, Hämatothorax nach Pleurapunktion $(n=1)$, septischer Schock im Rahmen eines lleus $(n=1)$, erwarteter Tod aufgrund von hohem Alter und schlechtem Allgemeinzustand entweder zu Hause oder in einer Pflegeeinrichtung $(n=8)$, unbekannt $(n=3)$. 
Todesursachen

Katz $=6$

$(n=156)$

Prozedurale Mortalität (VARC-Definition) $(n=43)$

Unmittelbar Prozedurbedingt:

- Aortenwurzelruptur

- Schlaganfall

- Kardiogener Schock

Infektionen:

- Pneumonie

- Septisch-Kardiogener Schock/ SIRS

- Pleura-Empyem

Arrhythmien:

- Plötzlicher Herztod

- Dokumentierte Ventrikuläre Arrhythmie

Blutungskomplikationen:

- hämorrhagischer Schock

- Herzbeuteltamponade

- Gastrointestinale Blutung

Andere:

- Mesenteriale Ischämie

- Aspiration

Todesfälle im Follow-Up-Zeitraum $(n=74)$

Kardiale Ursachen:

Manifeste Herzinsuffizienz

Plötzlicher Herztod

Zugangsbedingte Mortalität:

Schlaganfall

Andere Gefäßkomplikationen (periphere

Embolisationen, nachfolgende Sepsis)

Infektionen:

Pneumonie

Septischer Schock mit unklarem Fokus

Herzklappenendokarditis

Septischer Schock im Rahmen eine lleus

Malignom

Andere Eingriffe (z.B. wg. Schenkelhalsfraktur)

Blutungskomplikationen:

Gastrointestinale Blutung

Haematothorax nach Pleurapunktion

Akutes Nierenversagen

Altersschwäche / schlechter AZ

Unbekannte Ursachen
$10(6.4 \%)$

$5(3.2 \%)$

0

$3(1.9 \%)$

$2(1.3 \%)$

$4(2.6 \%)$

$1(0.6 \%)$

$3(1.9 \%)$

0

0

0

0

0

0

0

0

$1(0.6 \%)$

0

$27(17.3 \%)$

$13(8.3 \%)$

$9(5.8 \%)$

$4(2.6 \%)$

$2(1.3 \%)$

$1(0.6 \%)$

$1(0.6 \%)$

$3(1.9 \%)$

$2(1.3 \%)$

0

$1(0.6 \%)$

0

$4(2.6 \%)$

$1(0.6 \%)$

\section{0}

0

0

$1(0.6 \%)$

$1(0.6 \%)$

$2(1.3 \%)$
Katz $<6$

( $n=144)$

$\mathbf{P}$

$33(23 \%) \quad<0.0001^{\star}$

$14(9.7 \%)$

$0.02^{*}$

$2(1.4 \%)$

0.14

$6(4.2 \%)$

0.26

$6(4.2 \%)$

0.12

$10(6.9 \%)$

0.07

$5(3.5 \%)$

0.08

$4(2.8 \%)$

0.62

$1(0.7 \%)$

0.30

$4(2.8 \%)$

$0.04^{*}$

$2(1.4 \%)$

0.14

$2(1.4 \%)$

0.14

$4(2.8 \%)$

$0.04^{\star}$

$2(1.4 \%)$

0.14

$1(0.7 \%)$

0.30

$1(0.7 \%)$

0.30

0.34

0.30

$1(0.7 \%)$

$0.002^{*}$

0.17

0.52

0.19

$8(5.6 \%)$

0.61

$1(0.7 \%)$

0

0.34

$1(0.7 \%)$

0.95

$10(6.9 \%)$

$0.03^{*}$

$4(2.8 \%)$

0.36

$4(2.8 \%)$

$0.04^{*}$

$1(0.7 \%)$

0.95

$1(0.7 \%)$

0.30

$2(1.4 \%)$

0.47

$4(2.8 \%)$

0.15

$3(2.1 \%)$

0.07

$2(1.4 \%)$

0.14

$1(0.7 \%)$

0.30

0.34

$0.02^{\star}$

0.61

Tabelle 3: Todesursachen bei gebrechlichen und nicht-gebrechlichen Patienten 
Zusätzlich führten wir Überlebenszeitanalysen durch, um die Vorhersagequalität verschiedener basisdemographischer Parameter, Risikoscores und des Gebrechlichkeitsstatus im Hinblick auf die Langzeitmortalität zu überprüfen. In univariaten Analysen identifizierten wir so die folgenden Parameter als signifikante Prädiktoren der Mortalität: den transapikalen Zugangsweg, ein NYHA-Stadium IV vor TAVI, eine reduzierte linksventrikuläre Ejektionsfraktion (EF $<55 \%$ ), ein pulmonalarterieller systolischer Druck von $\geq 44.5 \mathrm{mmHg}$, eine reduzierte glomeruläre Filtrationsrate (GFR) $<30 \mathrm{~mL} / \mathrm{min} / 1.73 \mathrm{~m}^{2} \mathrm{bzw}$. auch $<60 \mathrm{~mL} / \mathrm{min} / 1.73 \mathrm{~m}^{2}$, Diabetes mellitus, präoperative Anämie mit HämoglobinWerten $<11.5 \mathrm{~g} / \mathrm{dl}$, alle drei chirurgischen Risikoscores sowie den Status der "Gebrechlichkeit" (definiert via Katz-Index, Barthel-Index, Modified Ranking Scale oder nach dem Vorschlag von Lee et al., vgl. auch Tabelle 4). Interessanterweise waren andere für die konventionelle Klappenchirurgie bekannte Risikofaktoren wie höheres Lebensalter, weibliches Geschlecht, vorangegangene Herzoperationen, pAVK sowie COPD nicht mit einer erhöhten Sterblichkeit assoziiert. Die Risikofaktoren die sich in den univariaten Analysen als signifikant erwiesen, wurden mittels multivariater Analyse (Cox Proportional Hazards Model) auf ihre Unabhängigkeit hin getestet. Da sich bei den gebrechlichen und nicht-gebrechlichen Individuen die Parameter "Alter" und "Geschlecht" signifikant unterschieden, wurden diese beiden Merkmale in die multivariate Analyse mit einbezogen. Aufgrund der Redundanz der verschiedenen geriatrischen Scores wurde der Katz-Index für die multivariate Analyse ausgewählt, da dieser in der univariaten Analyse die höchste Hazard Ratio aller Frailty Scores erzielte. Im multivariaten Modell kristallisierten sich schließlich nur vier Parameter als signifikante unabhängige Prädiktoren der Langzeitmortalität heraus: das NYHA-Stadium IV vor TAVI, eine GFR $<30$ $\mathrm{mL} / \mathrm{min} / 1.73 \mathrm{~m}^{2}$, Diabetes mellitus und ein Katz-Index $<6$ (vgl. Tabelle 3). Der Katz-Index $<6$ hatte unter diesen vier Parametern die höchste Hazard Ratio hinsichtlich der Mortalitätsprädiktion (2.67). Dagegen erwies sich keiner der chirurgischen Risikoscores als signifikanter unabhängiger Prädiktor der Langzeitmortalität. Schlussendlich stellte sich in unserem Patientenkollektiv als bedeutsamster Prädiktor der Langzeitmortalität somit das Merkmal "Gebrechlichkeit" (definiert als Einschränkung in den ATLs = Katz $<6$ ) heraus. 


\begin{tabular}{|c|c|c|c|c|}
\hline & \multicolumn{2}{|c|}{ Univariate Analyse } & \multicolumn{2}{|c|}{ Multivariate Analyse } \\
\hline & Hazard Ratio* & P [logrank] & Hazard Ratio* & $P$ [coxph] \\
\hline \multicolumn{5}{|l|}{ Risikofaktor } \\
\hline Alter $\geq 83$ Jahre (=Median) & $0.91[0.6-1.3]$ & 0.61 & $0.99[0.9-1.0]$ & 0.63 \\
\hline Weibliches Geschlecht & $0.83[0.6-1.2]$ & 0.35 & $1.46[0.9-2.3]$ & 0.10 \\
\hline $\mathrm{BMI} \geq 20 \mathrm{~kg} / \mathrm{m}^{2}$ & $0.96[0.4-2.7]$ & 0.93 & & \\
\hline KÖF $\geq 1.77 \mathrm{~m}^{2}$ (=Median) & $0.90[0.6-1.3]$ & 0.58 & & \\
\hline Transapikaler Zugangsweg & $1.59[1.1-2.3]$ & $0.01^{*}$ & $1.09[0.7-1.7]$ & 0.70 \\
\hline \multicolumn{5}{|l|}{ Kardiale Vorerkrankungen } \\
\hline NYHA-Stadium IV vor TAVI & 2.64 [1.8-3.9] & $<0.001^{*}$ & $1.73[1.1-2.7]$ & $0.02^{\star}$ \\
\hline KHK & $1.43[1.0-2.2]$ & 0.08 & & \\
\hline Z.n. PTCA & $1.31[0.9-1.9]$ & 0.17 & & \\
\hline Z.n. Bypassoperation & $1.25[0.8-2.0]$ & 0.38 & & \\
\hline Z.n. anderer Thorakotomie & $0.80[0.3-2.5]$ & 0.70 & & \\
\hline LV-EF $<55 \%$ vor TAVI & $1.47[1.0-2.1]$ & $0.04^{\star}$ & $0.97[0.6-1.5]$ & 0.88 \\
\hline PAPs $\geq 44.5 \mathrm{mmHg}$ (=Median) & $1.59[1.1-2.4]$ & $0.02^{*}$ & $1.38[0.9-2.1]$ & 0.15 \\
\hline $\mathrm{Al} \geq$ Grad I nach TAVI & $1.43[0.9-2.2]$ & 0.11 & & \\
\hline \multicolumn{5}{|l|}{ Nicht-kardiale Erkrankungen } \\
\hline Z.n. TIA oder Apoplex & $1.51[0.9-2.4]$ & 0.09 & & \\
\hline pAVK & $1.37[0.9-2.0]$ & 0.10 & & \\
\hline GFR $<30 \mathrm{~mL} / \mathrm{min} / 1,73 \mathrm{~m}^{2}$ & $3.40[2.3-5.1]$ & $<0.001^{*}$ & $2.22[1.3-3.8]$ & $0.003^{\star}$ \\
\hline GFR $<60 \mathrm{~mL} / \mathrm{min} / 1,73 \mathrm{~m}^{2}$ & $1.76[1.2-2.6]$ & $0.005^{\star}$ & & \\
\hline COPD & $1.07[0.7-1.6]$ & 0.75 & & \\
\hline Diabetes mellitus & $1.9[1.3-2.8]$ & $<0.001^{*}$ & $1.60[1.1-2.4]$ & $0.03^{\star}$ \\
\hline $\mathrm{Hb}<11.5 \mathrm{~g} / \mathrm{dL}$ (=Median) & $1.58[1.1-2.3]$ & $0.01^{*}$ & $0.96[0.6-1.5]$ & 0.86 \\
\hline \multicolumn{5}{|l|}{ Chirurgische Risikoscores } \\
\hline Log. EuroScore I $\geq 20 \%$ & $1.93[1.3-2.9]$ & 0.001 & & \\
\hline Log. EuroScore I $\geq 40 \%$ & $2.30[1.5-3.5]$ & $<0.001^{*}$ & $0.68[0.4-1.3]$ & 0.24 \\
\hline EuroScore $\| \mathrm{I} \geq 10 \%$ & $2.61[1.8-3.8]$ & $<0.001^{*}$ & $1.73[1.0-3.1]$ & 0.06 \\
\hline STS-Score $\geq 10 \%$ & $2.45[1.6-3.7]$ & $<0.001^{*}$ & $1.00[0.6-1.8]$ & 0.99 \\
\hline \multicolumn{5}{|l|}{ Geriatrische Scores } \\
\hline Katz-Index <6 (=Median) & $2.67[1.8-3.9]$ & $<0.001^{*}$ & $2.67[1.7-4.3]$ & $<0.0001^{\star}$ \\
\hline Barthel-Index <95\% (=Median) & $2.64[1.8-3.9]$ & $<0.001^{*}$ & & \\
\hline Modified Ranking Scale $\geq 2$ & $2.49[1.5-4.1]$ & $<0.001^{*}$ & & \\
\hline Gebrechlichkeit nach Lee et. al. & $2.34[1.6-3.5]$ & $<0.001^{*}$ & & \\
\hline
\end{tabular}

Tabelle 4: Prädiktoren der Langzeitmortalität in uni- und multivariaten Analysen ( ${ }^{*} 95 \%$ Konfidenzintervalle in Klammern dargestellt) 


\subsection{Der Einfluss der Gebrechlichkeit}

\subsubsection{Basisdemographische und prozedurale Charakteristika}

Verglichen mit der Gruppe der komplett selbständigen Patienten war die Gruppe der Patienten mit einem Katz-Index $<6$ durchschnittlich zwei Jahre älter $(P=0.0004)$, häufiger weiblich $(P=0.01)$ und wies häufiger eine Niereninsuffizienz mit einer GFR $<30 \mathrm{ml} / \mathrm{min} / 1.73 \mathrm{~m}^{2}$ auf $(P=0.04)$ (vgl. Tabelle 1). Weiterhin befanden sich diese Patienten in fortgeschritteneren Stadien der Herzinsuffizienz (NYHA-Stadium IV in $27 \%$ vs. $10 \%, P=0.0001$ ) und klinische Zeichen der Herzschwäche waren deutlich häufiger zu beobachten. Die präoperativen echokardiographischen Parameter (Transvalvuläre Gradienten, Ejektionsfraktion, Aortenklappenöffnungsfläche, pulmonalarterieller systolischer Druck sowie Prävalenz und Grad der Aortenklappeninsuffizienz) unterschieden sich hingegen nicht.

Bei Betrachtung der prozeduralen Parameter in beiden Gruppen waren keine Unterschiede hinsichtlich der Eingriffsdauer $(P=0.52)$, Fluoroskopie-Zeit $(P=0.92)$, oder des Kontrastmittelvolumens $(P=0.85)$ festzustellen.

\subsubsection{Intrahospitaler Verlauf}

Periprozedurale Komplikationen, Mortalitätsraten und Todesursachen sind für gebrechliche und nicht-gebrechliche TAVI-Patienten in den Tabellen 2 und 3 aufgeführt. Die einzige Komplikation mit signifikant unterschiedlicher Inzidenz in beiden Gruppen war das akute Nierenversagen im Stadium drei, was nicht überraschend ist, wenn man sich vor Augen führt, dass die Patienten mit einem Katz-Index $<6$ bereits vor der TAVI eine wesentlich schlechtere Nierenfunktion aufwiesen. Sämtliche andere schwerwiegenden Komplikationen waren dagegen in den beiden Gruppen gleichverteilt $(P=0.93$ für Schlaganfall, $P=0.95$ für Myokardinfarkt, $P=0.35$ für Verschlüsse der Koronarien, $P=0.60$ für lebensgefährliche Blutungen, $\mathrm{P}=0.56$ für schwerwiegende Gefäßkomplikationen). Dennoch waren die beobachteten Sterblichkeitsraten zu allen definierten Zeitpunkten signifikant höher in der Gruppe der gebrechlichen Patienten (5.5\% vs. $1.3 \%$ unmittelbar prozedurale Mortalität, $17 \%$ vs. $5.8 \% 30$-TageSterblichkeit und $23 \%$ vs. $6.4 \%$ prozedurale Mortalität). Diese Tatsache konnte 
damit erklärt werden, dass Patienten mit einem Katz-Index $<6$ bei Eintritt einer schwerwiegenden Komplikation signifikant häufiger an dieser verstarben. Beispielsweise verstarb niemand aus der Gruppe der selbständigen Patienten an Blutungen oder an Gefäßzugangskomplikationen, während bei den Gebrechlichen vier von fünfzehn Patienten (27\%) an einer Blutung und fünf von vierzehn Patienten $(36 \%)$ an Zugangskomplikationen verstarben ( $\mathrm{P}=0.02 \mathrm{im}$ Vergleich von beiden Gruppen). Ähnlich verhielt es sich mit prozedurbedingten Schlaganfällen. Nur drei von zehn selbständigen Patienten verstarben am erlittenen periprozeduralen Apoplex (30\%), während bei den Patienten mit einem Katz-Index $<6$ sechs von neun Patienten ihrem Schlaganfall erlagen (66\%) $(P=0.1)$. Auch die Pneumonie als Todesursache war häufiger bei Individuen mit einem Katz-Index $<6$ vertreten (fünf Patienten vs. ein Patient, $P=0.08$ ). Vergleicht man die auf den chirurgischen Risikomodellen basierenden 30Tages-Mortalitäts-Schätzwerte, so hatten die gebrechlichen Patienten gemäß allen Modellen signifikant erhöhte Werte (29\% vs. $24 \%$ für log. EuroSCORE I, $9.5 \%$ vs. $7.5 \%$ für den EuroSCORE II und $8.8 \%$ vs. $5.9 \%$ für den STSScore). Dennoch reflektierten diese Einschätzungen weder die tatsächliche 30Tages-Mortalität in beiden Gruppen (17\% vs. $5.8 \%$ ) noch das dreifach erhöhte Sterblichkeitsrisiko der gebrechlichen Patienten (siehe Abbildung 6).

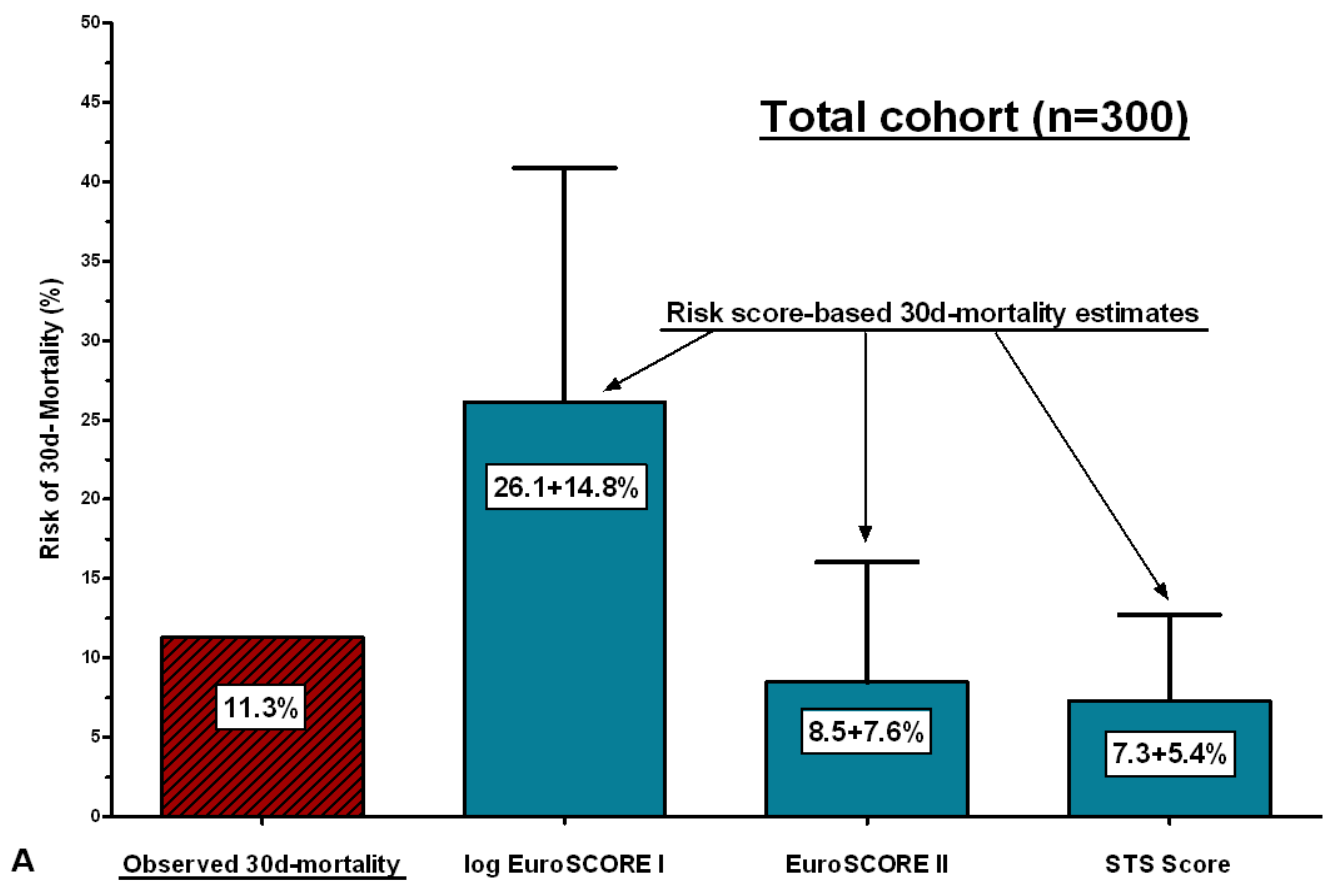



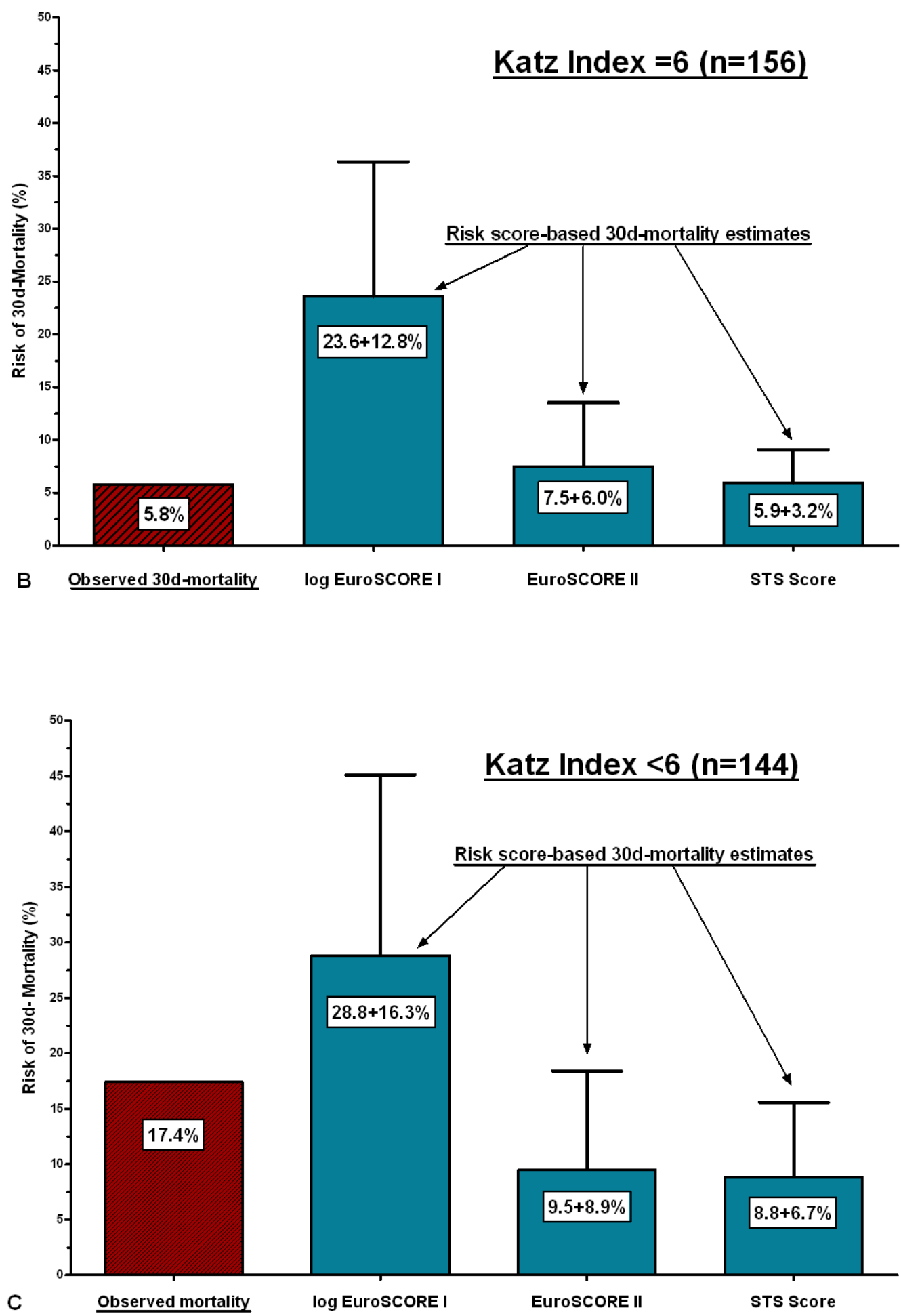

Abb. 6: Vergleich der mittels Risikoscores erhobenen Schätzwerte mit der tatsächlichen 30-Tages-Mortalität der TAVI-Patienten:
A) im Gesamtkollektiv
B) bei nicht-gebrechlichen Patienten
C) bei gebrechlichen Patienten 
Der VARC-definierte kombinierte Sicherheitsendpunkt war wesentlich häufiger bei gebrechlichen Patienten aufgetreten ( $40.3 \%$ vs. $24.4 \%, \mathrm{P}=0.003$ ), was vor allem auf die höhere 30-Tages-Mortalität und häufigere Inzidenz des akuten Nierenversagens zurückzuführen war. Darüber hinaus waren Patienten mit Einschränkungen in den ATLs einem signifikant höheren Risiko für prolongierte Krankenhausaufenthalte von mehr als zwei Wochen Dauer ausgesetzt (32\% vs. $22 \%, P=0.047$; der mediane Krankenhausaufenthalt betrug in dieser Gruppe 23 Tage). Zusätzlich wurden auch signifikant mehr Patienten mit einem initialen Katz-Index $<6$ nach durchgeführter TAVI in Pflegeeinrichtungen verlegt (59/111 vs. $13 / 146$ lebend entlassene Personen, $P<0.0001)$.

\subsubsection{Analyse von Morbidität und Mortalität im Langzeitverlauf}

Während der medianen Nachbeobachtungszeit von 537 Tagen starben insgesamt 117 Patienten, wobei der Anteil der Todesfälle unter den gebrechlichen Patienten deutlich höher lag. Verstorben waren 80 von 144 gebrechlichen Patienten (56\%, mediane Überlebenszeit 759 Tage) und $37 \mathrm{der}$ $156(24 \%)$ nicht-gebrechlichen Patienten $(P<0.0001)$ (siehe entsprechende Kaplan-Meier-Kurve Abb.7). Besonders bei Personen mit einem Katz-Index $<6$ und einer zusätzlich stark eingeschränkten GFR $<30 \mathrm{ml} / \mathrm{min} / 1.73 \mathrm{~m}^{2}$ war eine erhöhte Sterblichkeit zu beobachten. Dieses Patientenkollektiv erreichte nur ein medianes Überleben von 86.5 Tagen, und die Mortalitätsrate stieg in dieser Gruppe innerhalb von zwei Jahren auf nahezu $75 \%$.

Ebenfalls deutlich schlechter sah es in der gebrechlichen Gruppe auch beim ereignisfreien Überleben aus (medianes ereignisfreies Überleben von 395 vs. 888 Tagen bei selbständigen Patienten, $\mathrm{P}=0.002$ ), wobei diese Tatsache überwiegend auf die Todesfälle zurückzuführen war. Hingegen traten kardiovaskulär bedingte Rehospitalisierungen in beiden Gruppen gleich häufig auf. 


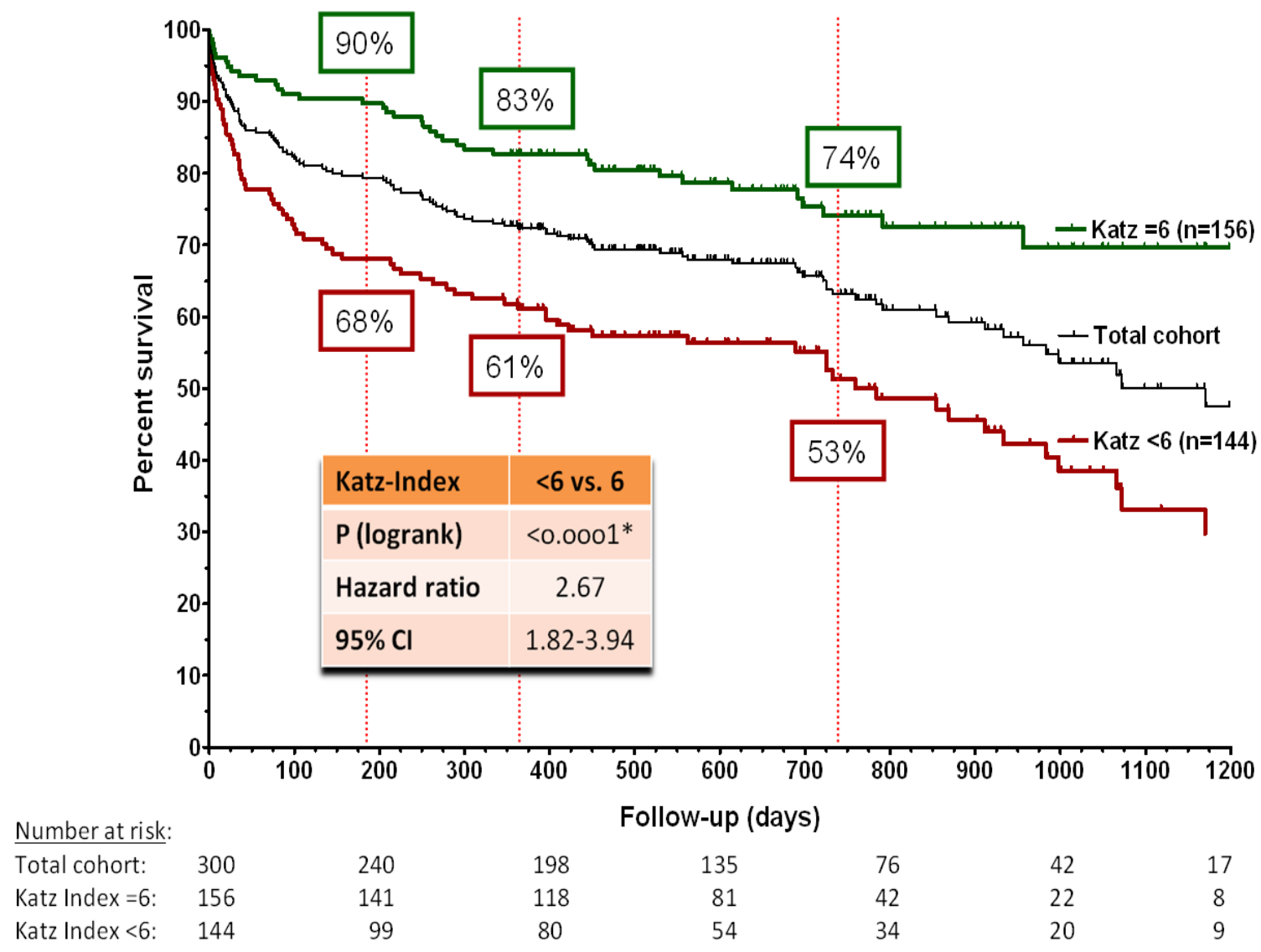

Abb. 7: Langzeitmortalität der gebrechlichen vs. nicht-gebrechlichen Patienten schwarze Kurve $\rightarrow$ in der gesamten Kohorte

grüne Kurve $\rightarrow$ bei nicht-gebrechlichen Patienten (Katz-Index $=6$ )

rote Kurve $\rightarrow$ bei gebrechlichen Patienten (Katz-Index $<6$ )

Nachfolgend führten wir eine Subgruppen-Analyse entsprechend des Schweregrades der Gebrechlichkeit durch. Hierfür wurde zwischen den zwei Subgruppen der schwer gebrechlichen (Katz-Index von 0-2) und der moderat gebrechlichen (Katz-Index von 3-5) Patienten differenziert. Die entsprechenden Kaplan-Meier-Kurven in Abb. 8 heben eindrucksvoll hervor, dass parallel mit zunehmenden Grad der Beeinträchtigung in den ATLs die Überlebensraten kontinuierlich abnahmen: das mediane Überleben der moderat gebrechlichen Subgruppe lag bei 998 Tagen im Vergleich zum medianen Überleben von 279 Tagen in der schwer gebrechlichen Subgruppe $(P=0.002)$. 


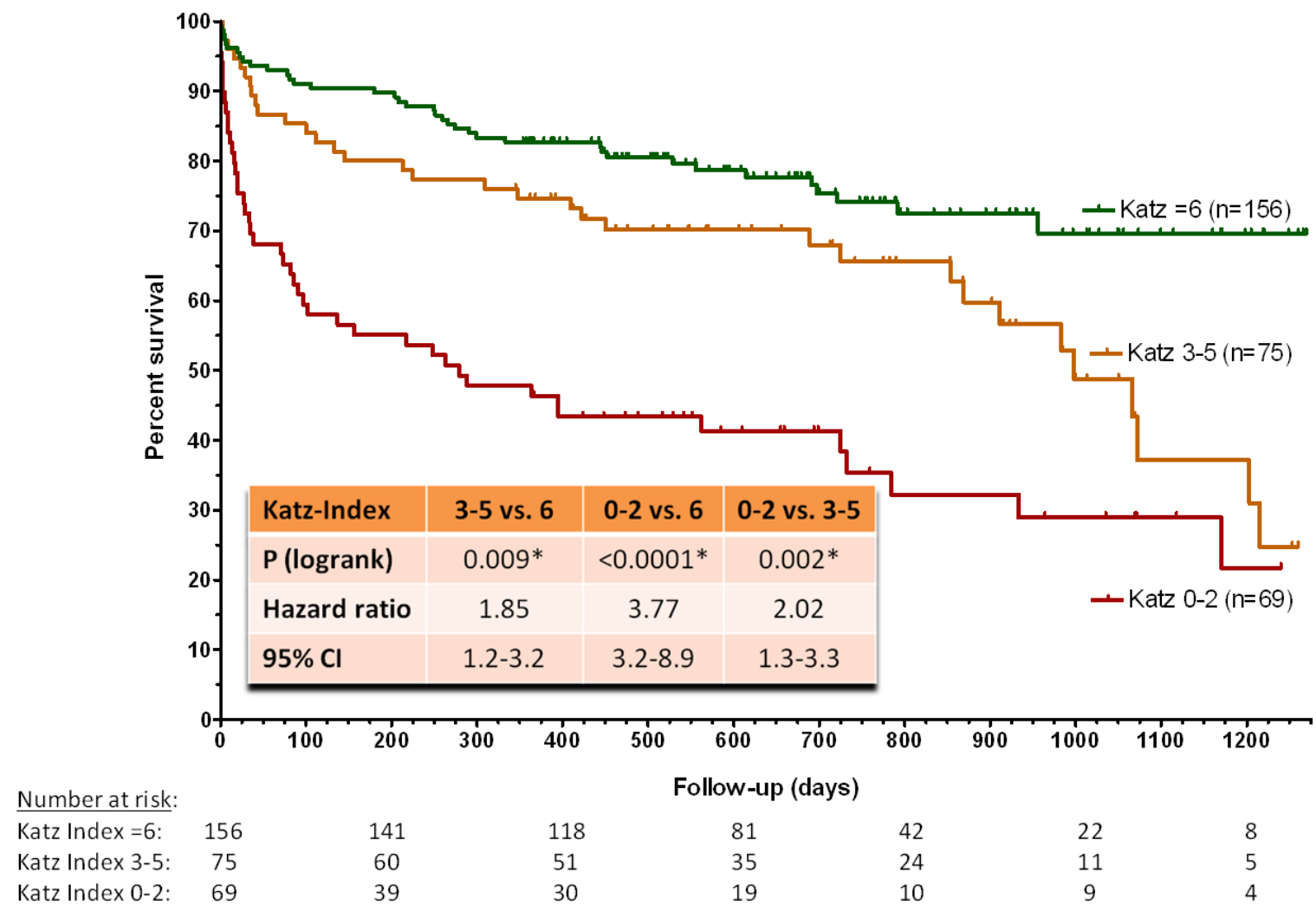

Abb. 8: Subgruppenanalyse zur Differenzierung der Langzeitmortalität zwischen den gebrechlichen Patienten mit Katz-Index 0-2 und Katz-Index 3-5

Um zu klären, ob der Gebrechlichkeitsstatus vorwiegend die kurzfristige oder die langfristige Mortalität beeinflusst, führten wir in einem zweiten Schritt eine Landmark-Analyse durch. Das Merkmal der Gebrechlichkeit, definiert als jegliche Form von Einschränkungen im Katz-Index, erwies sich sowohl als signifikanter Prädiktor der 30-Tages-Mortalität ( $\mathrm{P}=0.003$, HR 3.05, Abb. 9 - alle späteren Todesfälle zensiert) als auch der langfristigen Mortalität nach Tag 30 $(P<0.0001$, HR 2.50, Abb.10 - alle Todesfälle zw. Tag 0 und Tag 30 zensiert). Interessanterweise deckte die Subgruppen-Analyse auf, dass moderat Gebrechliche (Katz-Index 3-5) im Vergleich zu selbständigen Personen keine signifikant erhöhte 30-Tages-Mortalität aufwiesen ( $P=0.79)$, die schwer Gebrechlichen (Katz-Index 0-2) aber schon ( $P<0.0001$, HR 5.35; vgl. Abb. 9). Im Langzeitverlauf näherten sich die Mortalitätsraten der moderat gebrechlichen Patienten den Sterblichkeitsraten der schwer gebrechlichen Patienten an und differierten zum Ende hin nicht mehr signifikant voneinander ( $P=0.1, H R$ 1.56), 
so dass beide Gruppen eine im Vergleich zu selbständigen Patienten signifikant erhöhte Langzeitmortalität hatten (vgl. Abb. 10).

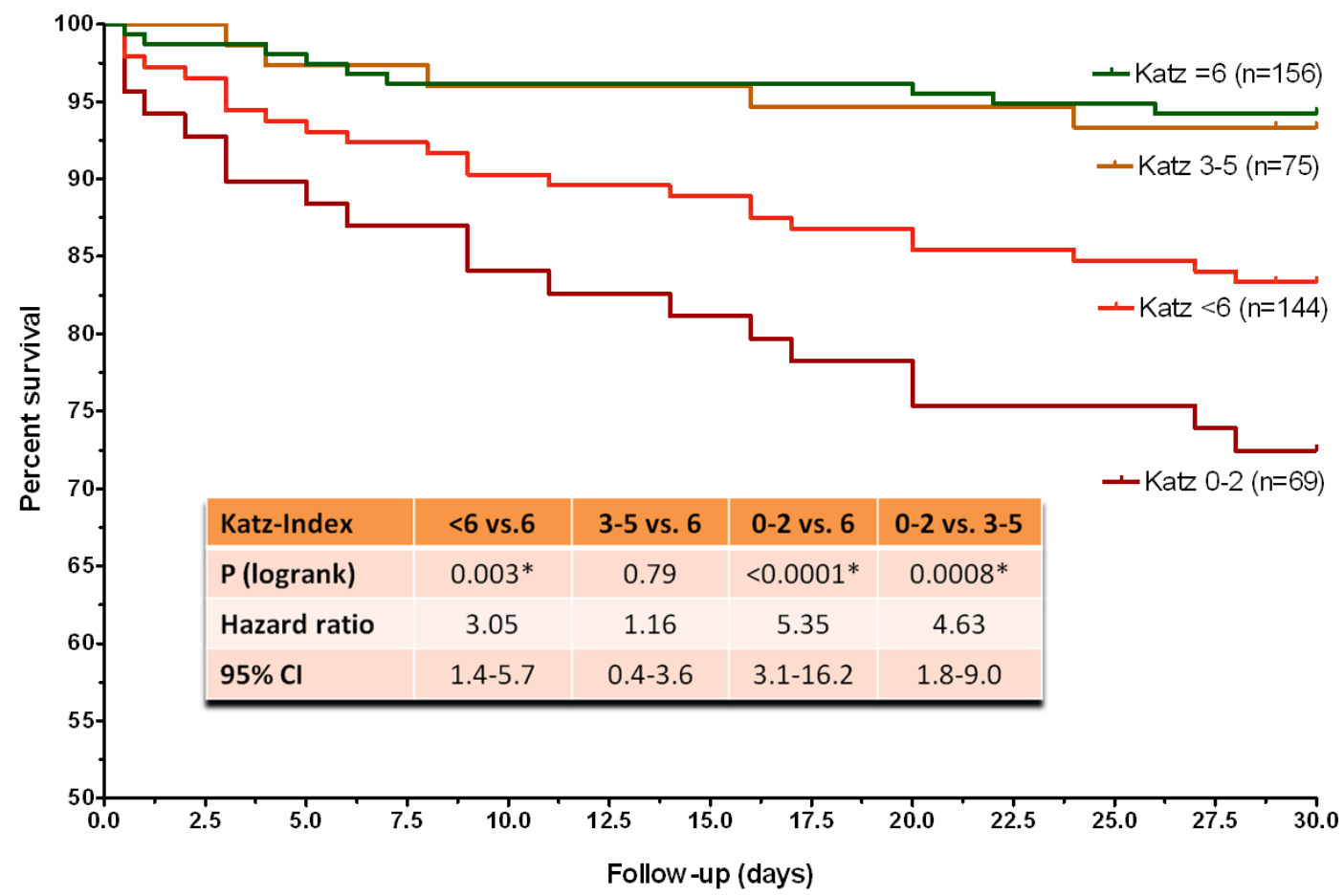

Abb. 9: Landmark-Analyse Tag 0-30 inkl. Subgruppen-Darstellung

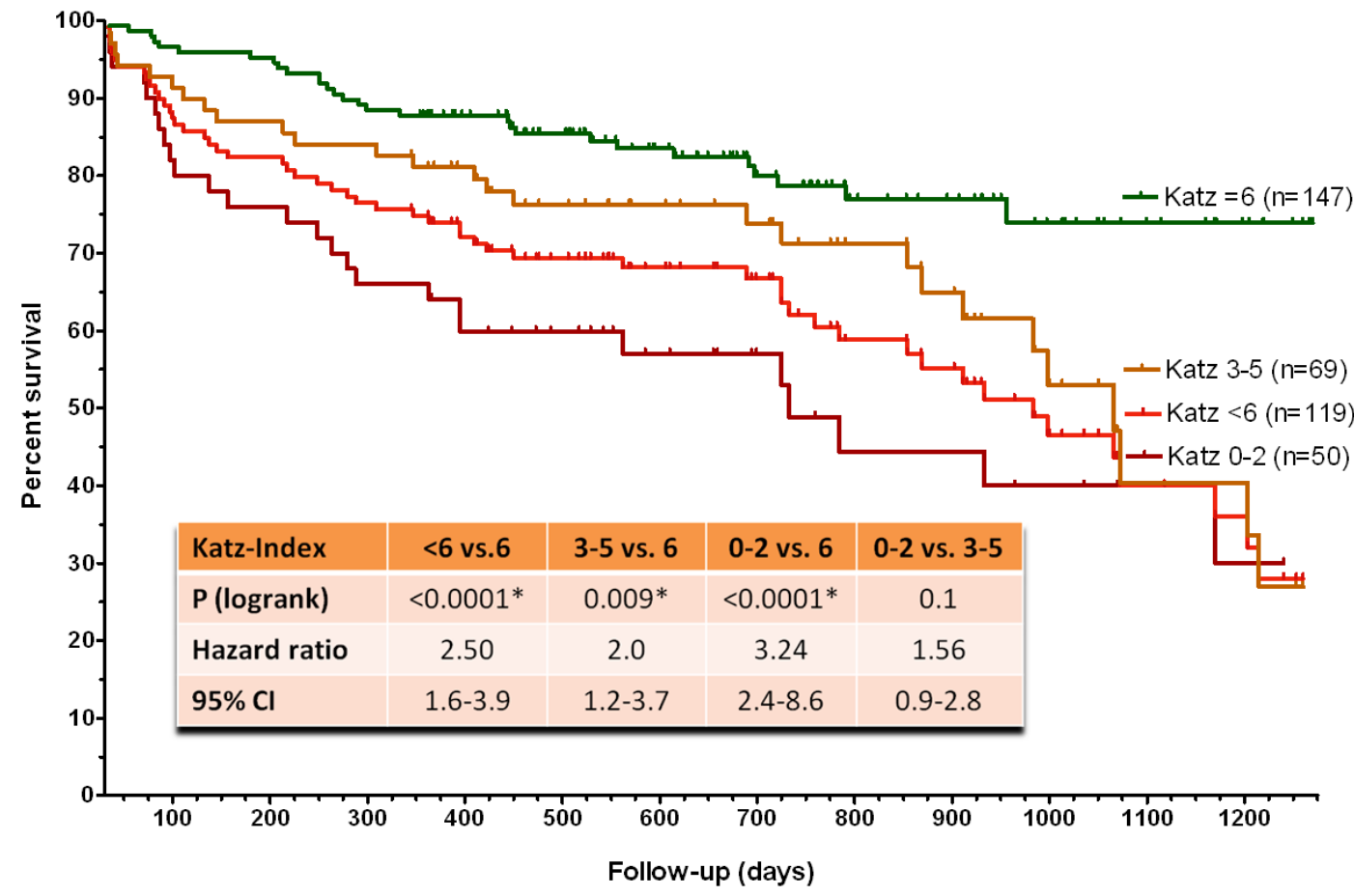

Abb. 10: Landmark-Analyse ab Tag 31 inkl. Subgruppen-Darstellung (Todesfälle bis zum Tag 30 zensiert) 


\subsection{NYHA-Status vor und nach TAVI}

Der überwiegende Teil der 300 Patienten aus unserem Kollektiv befand sich vor dem Eingriff in einem NYHA-Stadium $\geq$ III (93\%), wobei $18 \%$ der Patienten bereits ein NYHA-Stadium IV mit Dyspnoe im Ruhezustand aufwiesen. Nur $7 \%$ hatten präoperativ einen NYHA-Status $\leq$ II (siehe Abb. 11).

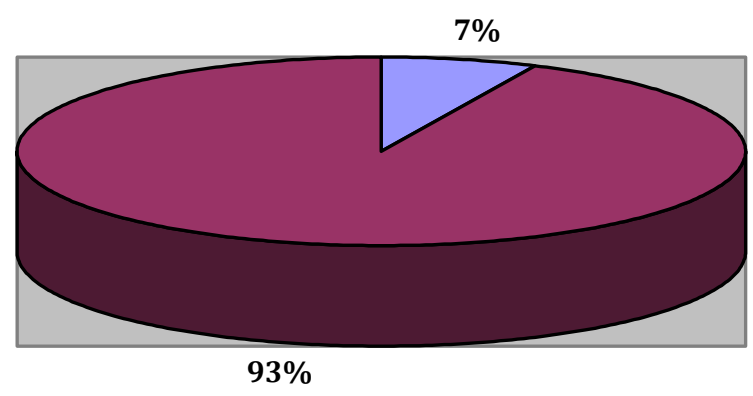

Abb. 11: NYHA-Status vor TAVI (300 Patienten)

Im Rahmen des telefonischen Follow-up wurde auch der postinterventionelle NYHA-Status erfragt. Es fiel auf, dass der Großteil der Patienten nach der TAVI eine deutliche Verbesserung der Dyspnoesymptomatik angab (siehe Abb. 12). 159 der Patienten (87\%) verspürten entweder keine Luftnot mehr oder lediglich eine geringe Dyspnoe bei stärkerer Belastung entsprechend einem NYHAStadium $\leq$ II. Bei 24 Patienten (13\%) lag noch ein NYHA-Stadium $\geq$ III vor.

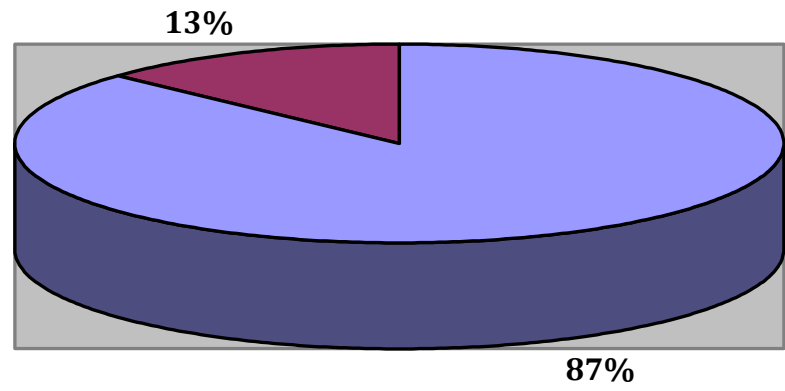

Abb. 12: NYHA-Status nach TAVI (183 Patienten) 


\subsection{Subjektive Beurteilung der Lebensqualität}

Hinsichtlich ihres langfristigen subjektiven Befindens gaben die überlebenden gebrechlichen Patienten signifikant seltener als nicht-gebrechliche Patienten eine Verbesserung ihrer Lebensqualität infolge des Eingriffes zu Protokoll (48\% vs. $64 \%, \mathrm{P}=0.04)$ - und dass, obwohl von einer positiven Selektion aufgrund etlicher Todesfälle vor allem unter den gebrechlichen Patienten auszugehen war.

Zum Zeitpunkt der letzten Befragung der noch lebenden 183 Patienten gaben insgesamt 156 Patienten an, dass es innen seit dem Eingriff besser gehe, 17 Patienten berichteten über ein gleichbleibendes subjektives Empfinden ihrer Lebensqualität und 10 Patienten fühlten sich seit dem Eingriff schlechter als vorher. Die letztgenannten wurden auch nach den Gründen für das subjektiv schlechtere Empfinden ihrer Lebensqualität befragt. Bei fünf der Patienten lag dies an Einschränkungen durch weitere Erkrankungen (Malignome, COPD oder beidseitige Unterschenkelamputation aufgrund einer $\mathrm{PAVK}$ ), drei Patienten gaben an, jetzt pflegebedürftiger als vor der TAVI zu sein (u. a. bei einem Patienten auch wegen eines erlittenen Apoplexes), ein weiterer Patient beklagte primär den Verlust des Ehepartners und Depressionen, und ein weiterer Patient litt aufgrund eines Mitralvitiums weiterhin an starker Dyspnoe. 


\section{Diskussion}

In Anbetracht des demographischen Alterns wird die steigende Inzidenz der degenerativen Aortenklappenstenose in der Zukunft ein weiter zunehmendes gesundheitsökonomisches Problem darstellen.

Für chirurgische Hochrisikopatienten, bei denen aufgrund von fortgeschrittenem Lebensalter und erheblichen Komorbiditäten ein konventionell chirurgischer Ersatz der Herzklappe abgelehnt wurde, steht seit gut einem Jahrzehnt mit der kathetergestützten Aortenklappenimplantation (TAVI) eine minimalinvasive Alternativtherapie zur Verfügung. In Übereinstimmung mit aktuellen Leitlinien (Vahanian et al. 2012) wurden an unserem Zentrum nur Patienten mit hochgradiger Aortenklappenstenose, die ein deutlich erhöhtes operatives Risiko aufwiesen, für eine TAVI vorgesehen. Während des Verlaufsbeobachtungszeitraumes dieses hochbetagten Patientenkollektives trat allerdings eine beachtenswerte Langzeitmortalität zu Tage: binnen der medianen Nachbeobachtungszeit von 537 Tagen starben insgesamt 117 der 300 TAVIPatienten.

In der vorliegenden Forschungsarbeit konnten wir das Merkmal "Gebrechlichkeit" (definiert als Verlust der Selbständigkeit in Aktivitäten des täglichen Lebens, Katz-Index < 6) als unabhängigen prognostischen Marker der kurz- und langfristigen Mortalität und Morbidität nach TAVI-Eingriffen identifizieren. Die Gruppe der gebrechlichen Patienten hatte während des primären stationären Aufenthaltes ein signifikant erhöhtes Risiko periprozedural $z u$ versterben, ungewöhnlich lange im Krankenhaus zu verweilen (> 14 Tage) sowie in Pflegeeinrichtungen entlassen zu werden. Auch während der weiteren Verlaufsbeobachtung hatten die gebrechlichen Patienten eine signifikant erhöhte Mortalität, während die selbständigen Patienten exzellente Langzeitüberlebensraten aufwiesen. Das über den Katz-Index definierte Merkmal der Gebrechlichkeit bestätigte sich in unserer Analyse als wichtigster unabhängiger Prädiktor der Langzeitmortalität. Hingegen zeigten alle in den Leitlinien empfohlenen chirurgischen Risikoscores keinen unabhängigen signifikanten Einfluss auf die Langzeitmortalität. 
Körperliche Gebrechlichkeit wird im Allgemeinen als geriatrisches Syndrom angesehen, welches mit einer verringerten Widerstandsfähigkeit des Organismus gegenüber Stressoren einhergeht und auf einen zunehmenden altersbedingten Verfall multipler physiologischer Systeme zurückzuführen ist. Daraus resultiert, dass gebrechliche Individuen in verstärktem Maße dem Risiko ausgesetzt sind, bereits bei geringfügigen Belastungen des Organismus zu versterben. Eine allgemeingültige Definition der Gebrechlichkeit oder eine weithin anerkannte Methode zur Messung derselben gibt es allerdings noch nicht.

Prinzipiell gibt es derzeit zwei verbreitete Sichtweisen des Merkmals der Gebrechlichkeit. Ein gebräuchliches Konzept definiert einen Phänotyp der Gebrechlichkeit anhand von fünf Kriterien: ungewollter Gewichtsverlust, Schwäche (per Handkraft im Hantelversuch gemessen), Ermüdung (mittels Fragebogen erfasst), Langsamkeit (ermittelt anhand der Zeit, die für eine 5Meter-Gehstrecke benötigt wird) sowie verminderte körperliche Aktivität (mit Fragebogen erfasst) (Fried et al. 2001). Die Befürworter dieses Konzeptes verlangen außerdem eine klare Differenzierung zwischen "Gebrechlichkeit", „funktioneller Behinderung“ (definiert als Einschränkungen in Alltagsaktivitäten) und "Komorbidität", welche in ihren Augen zwar drei miteinander verwandte aber dennoch prinzipiell unterschiedliche Sachverhalte darstellen (Fried et al. 2004).

Vertreter der konkurrierenden Betrachtungsweise hinterfragen dagegen den klinischen Nutzen dieser Aufteilung und messen Gebrechlichkeit einfach in Bezug auf die akkumulierten Defizite eines Individuums (Rockwood 2005). Das Bestreben dieses pragmatischeren Vorgehens ist es, Personen mit einem Risiko für einen voraussichtlich ungünstigeren klinischen Verlauf zu erkennen. Die Diskussion über die korrekte Definition der Gebrechlichkeit wirft eine zweite Frage auf: welche Methode eignet sich im klinischen Altag am besten zur Erfassung der Gebrechlichkeit? Eine ideale Messmethode sollte sowohl objektiv als auch reproduzierbar und im hektischen klinischen Altag möglichst einfach durchzuführen sein. Gleichzeitig sollte sie dabei helfen, Verbesserungsmöglichkeiten aufzuzeigen. Häufig wird Gebrechlichkeit allein mittels 
Blickdiagnose festgestellt, was weder objektiv noch reproduzierbar ist. Es existieren verschiedene geriatrische Scores zur Erfassung der Gebrechlichkeit, welche zwar objektiv, jedoch verhältnismäßig zeitaufwändig sind. Körperliche Belastungstests (wie der Hantelversuch) sind als Surrogatparameter ebenfalls objektiv und reproduzierbar, deuten dabei jedoch nicht zwangsläufig auf Interventionsmöglichkeiten hin, die zur Verbesserung der Ausgangslage führen könnten.

In diversen Publikationen wurde der Einfluss von Gebrechlichkeit auf Morbidität und Mortalität älterer Patienten thematisiert. Seit langem ist bekannt, dass der funktionelle Status eines Menschen, der gewöhnlich mit den Fähigkeiten zur Ausübung von Aktivitäten des täglichen Lebens erfasst wird, ein starker Prädiktor der Mortalität und Morbidität bei älteren Personen ist. Inouye et al. beobachteten, dass Einschränkungen in den ATLs bei über 70-jährigen allgemeinmedizinischen Patienten mit einem 1.9-fach erhöhten Risiko einhergingen, innerhalb von zwei Jahren zu versterben. Des Weiteren wurde inzwischen gezeigt, dass Gebrechlichkeit und das Eintreten von Abhängigkeiten in den Aktivitäten des täglichen Lebens (also Verlust der Selbständigkeit) stark miteinander assoziiert sind (Boyd et al. 2005).

Lee et al. untersuchten den Einfluss der Gebrechlichkeit auf die Mortalität in einer Population von 3826 herzchirurgischen Patienten (in dieser Studie wurden Personen mit einem Katz-Index $<6$, einer Gehbehinderung oder kognitiven Einschränkung als gebrechlich definiert). Während eines medianen Follow-up Zeitraumes von 1.8 Jahren erwies sich hierbei die Gebrechlichkeit als ein unabhängiger prognostischer Marker sowohl für die unmittelbar prozedurbedingte Mortalität (HR 1.8) als auch für die gesamte Lebenserwartung (HR 1.5). In Übereinstimmung mit Lees Beobachtungen etablierten Afilalo et al. die Zeitspanne zum Zurücklegen einer 5-Meter-Gehstrecke als weiteren bedeutsamen Prädiktor der eingriffsbedingten Mortalität und Morbidität in 131 älteren herzchirurgischen Patienten. Eine nachfolgende Untersuchung derselben Autoren (Afilalo et al. 2012) kombinierte die gängigen chirurgischen 
Risikoscores mit verschiedenen Stufen von Gebrechlichkeit und Behinderung, um auf diese Weise mögliche Hochrisikopatienten noch besser zu identifizieren.

Im Rahmen weiterer Untersuchungen mehren sich auch die Hinweise, dass die Evaluation von Gebrechlichkeit oder funktioneller Behinderung einen wertvollen Beitrag zur verbesserten Risikostratifizierung vor TAVI leisten kann. Unlängst wurde im Rahmen eines Screenings von 102 potentiellen TAVI-Patienten das Auftreten einer gebrechlichkeitsassoziierten Langsamkeit beim Überwinden der 5-Meter-Distanz eruiert (Green et al. 2012a). In dieser Studie trat bei $63 \%$ der Patienten eine deutlich verlangsamte Fortbewegung auf, welche darüber hinaus mit eingeschränkter Selbstversorgung verbunden war (dies wurde anhand des Verlustes der Selbständigkeit in den ATLs festgestellt). Auch der präinterventionelle 6-Minuten-Gehtest erwies sich als ein unabhängiger Prognosemarker in einer Kohorte von 260 konsekutiven TAVI-Patienten (HR 1.08 für jeden Verlust einer 10-Meter-Strecke, $P=0.001$ ) (Mok et al. 2013).

Zwei aktuelle Studien fokussieren sich auf den Einfluss von Gebrechlichkeit auf die Mortalität nach TAVI. Stortecky et al. konnten für 100 konsekutive TAVIPatienten in einem multidimensionalen Modell zeigen, dass alle erhobenen Parameter des geriatrischen Assessments (neben den ATLs auch Kognition, Ernährungszustand und Mobilität) signifikant mit der Mortalität nach 30 Tagen und nach einem Jahr nach TAVI assoziiert waren. Green et al. untersuchten prospektiv an 159 potentiellen TAVI-Kandidaten einen selbst entworfenen Gebrechlichkeitsindex, welcher die 5-Meter-Gehstreckenzeit, die Handkraft, das Serumalbumin und die Unabhängigkeit in den ATLs beeinhaltete. Nach dieser Definition als gebrechlich definierte Patienten verzeichneten tatsächlich eine erhöhte 1-Jahres-Sterblichkeit nach TAVI (Green et al. 2012b). Ergänzend zu dieser Beobachtung identifizierten Rodes-Cabau et al. die Gebrechlichkeit als einen unabhängigen Prognosefaktor der 2-Jahres-Sterblichkeit nach TAVI (Studie mit 339 Patienten), wobei diese jedoch ausschließlich mittels Blickdiagnose erfasst wurde.

In der vorliegenden Arbeit war es uns möglich, den Einfluss der Gebrechlichkeit (definiert als Verlust der Selbständigkeit in den ATLs; Katz-Index <6) als 
unabhängigen Prädiktor auf kurz- und langfristige Morbidität und Mortalität erstmals in einer großen Kohorte konsekutiver TAVI-Patienten zu belegen. Nach weitverbreiteter geriatrischer Ansicht wird durch Einschränkungen in den ATLs nicht die "Gebrechlichkeit" an sich sondern eine „Funktionseinschränkung" einer Person definiert. Manche Komponenten der gängigen phänotypischen Definition von Gebrechlichkeit (Fried et al. 2001) konnten jedoch nicht dazu beitragen, akkurat in unserer Patientenkohorte zu diskriminieren. Beispielsweise waren ein niedriger BMl und eine geringe Körperoberfläche kein Hinweis auf ein erhöhtes Sterblichkeitsrisiko: ein Umstand, der auf den bekannten Fakt rückführbar ist, dass bei adipösen Personen das Körpergewicht nicht unbedingt mit einem altersbedingten Verlust an Muskelmasse korrelieren muss (Rockwood 2005).

Vorangegangene Studien haben bereits einen starken Zusammenhang zwischen anerkannten Parametern von "Gebrechlichkeit" (z. B. Gehstrecke) und "Funktionseinschränkung" (gemessen durch Abhängigkeiten in den ATLs) aufgezeigt (Boyd et al. 2005, Green et al. 2012a). Daher wurde in der vorliegenden Untersuchung der Katz-Index zur Definition von "Gebrechlichkeit" anstelle von "Funktionseinschränkung" herangezogen, auch weil üblicherweise in der TAVI-relevanten Literatur nur von "Gebrechlichkeit" und nicht von „Funktionseinschränkung“ oder „Behinderung“ gesprochen wird und die Unterscheidung zwischen diesen Begriffen mehr eine Frage der Nomenklatur als der inhaltlichen Bedeutung $z u$ sein scheint. Der von Lee et al. veröffentlichte Vorschlag zur Definition von Gebrechlichkeit, in welchem zusätzlich zum Verlust von Fähigkeiten in den ATLs auch Gehbehinderung und Demenz berücksichtigt werden, führte in unserer Patientenkohorte zu einer schlechteren Risikostratifizierung mit niedrigerer Hazard-Ratio (HR 2.34, $\mathrm{P}<0.0001$ ) im Vergleich zur alleinigen Definition mit Hilfe des Katz-Index (HR 2.67, $\mathrm{P}<0.0001)$. Deshalb definierten wir Gebrechlichkeit letzten Endes als jedwede Form der Einschränkung in Aktivitäten des täglichen Lebens mit einem Katz-Index $<6$.

Der Katz-Index stellt einen sehr einfach bestimmbaren, reproduzierbaren und objektiven Risikoscore dar, der sich auch leicht im ruhelosen Klinikalltag 
anwenden lässt und dabei trotzdem eine zuverlässige Risikostratifizierung ermöglicht. Einschränkend muss darauf hingewiesen werden, dass in der vorliegenden Studie die Aussagekraft der ebenfalls gut bei kardialen Patienten diskriminierenden 5-Meter-Gehstrecke bzw. des 6-Minuten-Gehtestes nicht untersucht wurden.

Schlussfolgernd leiten wir aus unseren Studienergebnissen drei Konsequenzen $a b$ :

Interventionsmöglichkeiten, mit deren Hilfe sich der funktionelle Status einer Person verbessern lässt (wie beispielsweise Physiotherapie und Ergotherapie) könnten schon vor dem Eingriff bei gebrechlichen Patienten zum Einsatz kommen und so für eine positivere Ausgangslage vor Durchführung einer TAVI sorgen. In weiteren Studien sollte geklärt werden, inwieweit sich mit diesen Maßnahmen auch das langfristige Überleben nach TAVI verbessern lässt.

Weiterhin wird deutlich, dass eine kathetergestützte Aortenklappenimplantation idealerweise zu einem frühen Zeitpunkt, also noch vor dem Eintritt des Verlustes der Selbständigkeit, erfolgen sollte, um ein verbessertes Langzeitüberleben sicherzustellen. Sogar ein Screening noch nicht symptomatischer älterer Patienten auf eine Aortenklappenstenose hin wäre denkbar, da Menschen mit symptomatischer AS bekanntermaßen nur noch eine mediane Überlebenszeit von weniger als 2 Jahren haben (Horstkotte und Loogen 1988) und nach dem Auftreten von Symptomen in Verbindung mit Gebrechlichkeit auch nach Durchführung einer TAVI das Überleben nicht überzeugend verbessert wird. Bislang konnte keine Studie für diese spezielle gebrechliche Patientengruppe einen Überlebensvorteil nach kathetergestützter Aortenklappenimplantation belegen.

In einer Studie von Ben-Dor et al. wurden 274 inoperable Hochrisiko-Patienten (medianer log. EuroScore $42.4 \%$, STS-Score 12.8\%) mit hochgradiger AS nachverfolgt, welche nicht mittels TAVI, sondern medikamentös und per Ballonvalvuloplastie behandelt wurden. Die Überlebensraten in dieser Patientenkohorte lagen bei $68 \%$ nach sechs Monaten, $60 \%$ nach einem Jahr und $47 \%$ nach zwei Jahren. Diese Überlebensraten sind beinahe identisch mit 
den Überlebensraten unserer gebrechlichen Patientenkohorte nach der TAVIProzedur, und dies obwohl unsere eigenen Patienten weniger Komorbiditäten vorwiesen (Überlebensraten $68 \%$ nach sechs Monaten, 61 \% nach einem Jahr und $53 \%$ nach zwei Jahren).

In letzter Konsequenz sollte also gebrechlichen Patienten vor einer TAVI auch mitgeteilt werden, dass man in ihrem spezifischen Fall nicht sicher sagen kann, ob sie von dem Eingriff profitieren werden.

In der vorliegenden Arbeit wurde deutlich, dass der Einsatz des Katz-Index zur Bestimmung der Gebrechlichkeit eine verlässliche und nützliche Risikoabschätzung vor TAVI ermöglicht, während die empfohlenen chirurgischen Risikoscores in unserer Patientenkohorte unter mangelnder Kalibrierung leiden (wie z. B. Überschätzung der 30-Tage-Mortalität um das 4fache bei nicht gebrechlichen Patienten durch den log. EuroSCORE I oder Unterschätzung von fast $100 \%$ durch den STS Score bei Gebrechlichen). Der Nutzen dieser in den Leitlinien genannten Scores sollte deswegen im Zusammenhang mit TAVI-Patienten kritisch hinterfragt werden. Im Gegensatz dazu kann die Bestimmung der Gebrechlichkeit mittels des Katz-Index überzeugendere Vorhersagen treffen: Die Patientengruppe ohne Einschränkungen in den ATLs (Katz-Index $=6$ ) ist eine Gruppe mit exzellentem Langzeitüberleben, während gebrechliche Personen im Langzeitverlauf beachtlich schlechtere Überlebensraten aufweisen.

Im Kontext einer stetig steigenden Lebenserwartung und explodierender Kosten im Gesundheitswesen ist es wichtig, solche Patienten zu identifizieren, die von der kostenintensiven kathetergestützten Aortenklappenimplantation langfristig auch tatsächlich profitieren können. Zusammenfassend empfehlen wir, den Katz-Index in die Risikostratifizierung vor TAVI zu integrieren und den Eingriff, wenn möglich, frühzeitig und bei noch erhaltener oder wiederhergestellter Selbständigkeit der Patienten durchzuführen. 


\section{Zusammenfassung}

Hintergrund: Für ältere multimorbide Patienten stellt die kathetergestützte Aortenklappenimplantation (TAVI) eine deutlich weniger invasive Alternative zum offen chirurgischen Klappenersatz dar. Sowohl die mittel- als auch die langfristige Mortalität der TAVI-Patienten ist derzeit jedoch beachtlich. Deshalb untersuchten wir den Einfluss der Gebrechlichkeit auf die Kurz- und Langzeitmortalität nach TAVI mit Hilfe des Katz-Index.

Material und Methoden: Die vorliegende Untersuchung erfasst die ersten 300 konsekutiven Patienten (Durchschnittsalter $82 \pm 5$ Jahre), die eine TAVI in unserem Hause erhielten. 158 Patienten wurden über den transapikalen und 142 Patienten über den transfemoralen Zugangsweg behandelt.

Ergebnisse: 144 dieser Patienten waren präoperativ in mindestens einer ATL eingeschränkt und dementsprechend als gebrechlich definiert (Katz-Index $<6$ ). Mit Ausnahme der akuten Nierenschädigung vom Grad 3 waren alle ernsthaften postinterventionellen Komplikationen gleichmäßig in beiden Gruppen verteilt. Die peri-interventionelle Mortalität war jedoch bei den gebrechlichen Personen signifikant höher (5.5\% vs. $1.3 \%$ unmittelbar prozedurale Mortalität, $17 \%$ vs. $5.8 \% 30$-Tage-Mortalität, $23 \%$ vs. $6.4 \%$ prozedurale Mortalität). Die mittels chirurgischer Risikoscores geschätzte 30-Tage-Sterblichkeit (29\% vs. $24 \%$ für log. EuroSCORE I, $9.5 \%$ vs. $7.5 \%$ für EuroSCORE II, und $8.8 \%$ vs. $5.9 \%$ für STS-Score) reflektierte dabei weder die beobachtete 30-Tage-Mortalität beider Gruppen noch die generelle dreifache Risikoerhöhung bei gebrechlichen Patienten.

Der Katz-Index von $<6$ konnte weiterhin mittels multivariater Analyse als ein hochsignifikanter unabhängiger Prädiktor der Langzeitmortalität bestätigt werden (HR 2.67, P<0.0001): Während einer medianen Nachbeobachtung von 537 Tagen starben $55 \%$ der gebrechlichen Patienten im Vergleich zu $24 \%$ der nicht gebrechlichen Patienten. Dagegen hatte keiner der chirurgischen 
Risikoscores einen signifikanten unabhängigen Einfluss auf das Langzeitüberleben.

Schlussfolgerung: Der mit Hilfe des Katz-Index ermittelte GebrechlichkeitsStatus stellt einen starken prognostischen Faktor eines ungünstigen Verlaufes nach TAVI dar. Demgegenüber zeigten die in den Leitlinien empfohlenen chirurgischen Risikoscores eine mangelnde Kalibrierung hinsichtlich der 30Tage-Mortalität und ermöglichten darüber hinaus keine Aussage zum Langzeitüberleben. Zur Risikoabschätzung vor TAVI sollte daher der Katz-Index berücksichtigt werden, mit dessen Hilfe sich eine Patientengruppe mit sehr gutem Langzeitüberleben identifizieren lässt, die in besonderem Maße von einer TAVI profitiert. 


\section{Anhänge}

6.1 Anhang 1: Evaluationsbogen Aortenklappenstenose

\section{Evaluation der Aortenklappenstenose}

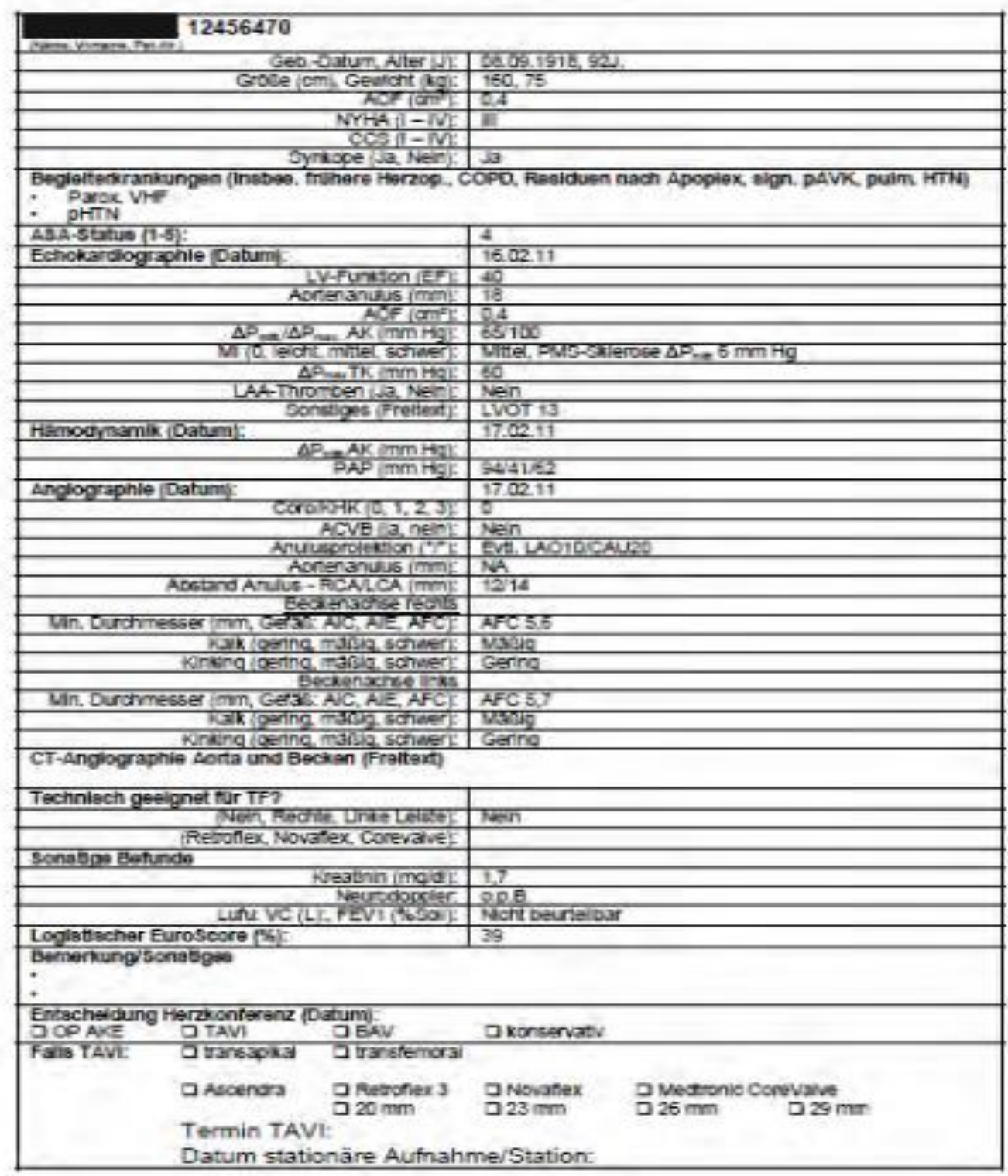


6.2 Anhang 2: Barthel-Index

\section{Aktivitäten des täglichen Lebens (ADL), Barthel-Index}

\section{Datum:}

\begin{tabular}{|c|c|}
\hline 1. Essen & Punikte \\
\hline Unabhängig, benutzt Geschirr und Besteck & 10 \\
\hline Braucht Hilfe, z.B. beim Schneiden & 5 \\
\hline Total hilfsbedürftig & 0 \\
\hline \multicolumn{2}{|l|}{ 2. Baden } \\
\hline Badet oder duscht ohne Hilfe & 5 \\
\hline Badet oder duscht mit Hilfe & 0 \\
\hline \multicolumn{2}{|l|}{ 3. Waschen } \\
\hline Wäscht Gesicht, kämmt, rasiert bzw. schminkt sich, putzt Zähne & 5 \\
\hline Braucht Hilfe & 0 \\
\hline \multicolumn{2}{|l|}{ 4. An- und Auskleiden } \\
\hline Unabhängig, inkl. Schuhe anziehen & 10 \\
\hline Hilfsbedürftig, kleidet sich teilweise selbst an/aus & 5 \\
\hline Total hilfsbedürftig & 0 \\
\hline \multicolumn{2}{|l|}{ 5. Stuhlkontrolle } \\
\hline Kontinent & 10 \\
\hline Teilweise inkontinent & 5 \\
\hline Inkontinent & 0 \\
\hline \multicolumn{2}{|l|}{ 6. Urinkontrolle } \\
\hline Kontinent & 10 \\
\hline Teilweise inkontinent & 5 \\
\hline Inkontinent & 0 \\
\hline \multicolumn{2}{|l|}{ 7. Toilettenbenutzung } \\
\hline Unabhängig bei Benutzung der Toilette/des Nachtstuhls & 10 \\
\hline Braucht Hilfe für z.B. Gleichgewicht, Kleidung aus-/anziehen, Toilettenpapier & 5 \\
\hline Kann nicht auf Toilette/Nachtstuhl & 0 \\
\hline \multicolumn{2}{|l|}{ 8. Bett-I(Roll-)Stuhltransfer } \\
\hline Unabhängig (gilt auch für Rollstuhlfahrer) & 15 \\
\hline Minimale Assistenz oder Supervision & 10 \\
\hline Kann sitzen, braucht für den Transfer jedoch Hilfe & 5 \\
\hline Bettlāgerig & 0 \\
\hline \multicolumn{2}{|l|}{ 9. Bewegung } \\
\hline Unabhängiges Gehen (auch mit Gehhilfe) für mindestens $50 \mathrm{~m}$ & 15 \\
\hline Mindestens $50 \mathrm{~m}$ Gehen, jedoch mit Unterstützung & 10 \\
\hline Für Rollstuhlfahrer: unabhängig für mindestens $50 \mathrm{~m}$ & 5 \\
\hline Kann sich nicht mindestens $50 \mathrm{~m}$ fortbewegen & 0 \\
\hline 10. Treppensteigen & \\
\hline Unabhängig (auch mit Gehhilfe) & 10 \\
\hline Braucht Hilfe oder Supervision & 5 \\
\hline Kann nicht Treppensteigen & 0 \\
\hline \multicolumn{2}{|l|}{ Gesamtpunktzahl (max. 100) } \\
\hline
\end{tabular}




\subsection{Anhang 3: EuroSCORE II}

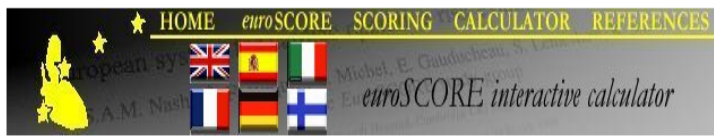

Important The previous additive ${ }^{1}$ and logistic ${ }^{2}$ EuroSCORE models are out of date. A new model has been prepared from fresh data and is launched at the 2011 EACTS meeting in Lisbon. The model is called EuroSCORE $\|^{3}$. this online calculator has been updated to use this new model. If you need to calculate the older "additive" or "logistic" EuroSCORE please visit the old calculator by clicking here

\begin{tabular}{|c|c|c|c|c|c|}
\hline \multicolumn{3}{|c|}{ Patient related factors } & \multicolumn{3}{|c|}{ Cardiac related factors } \\
\hline $\operatorname{Age}^{1}$ (years) & 75 & 0.46 & NYHA & ||l| $\checkmark$ & 2958358 \\
\hline Gender & female - & .2196434 & CCS class 4 angina ${ }^{8}$ & no - & 0 \\
\hline 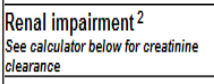 & moderate $(C C>50 \&<85)$ - & .303553 & LV function & moderate (LVEF 31\%-50\%) & .3150652 \\
\hline Extracardiac arteriopathy ${ }^{3}$ & yes. & .5360268 & Recent MII ${ }^{9}$ & no. & 0 \\
\hline Poor mobility ${ }^{4}$ & no - & 0 & Pulmonary hypertension 10 & no & 0 \\
\hline Previous cardiac surgery & yes. & 1.118599 & \multicolumn{3}{|c|}{ Operation related factors } \\
\hline Chronic lung disease ${ }^{5}$ & no - & 0 & Urgency ${ }^{11}$ & elective - & 0 \\
\hline Active endocarditits 6 & no. & 0 & Weight of the intervention 12 & 2 procedures & .5521478 \\
\hline Critical preoperative state ${ }^{7}$ & no - & 0 & Surgery on thoracic aorta & no - & 0 \\
\hline Diabetes on insulin & yes - & .3542749 & & & \\
\hline $\begin{array}{l}\text { EuroSCORE } \| \cdot \checkmark \\
\text { EuroSCORE }\end{array}$ & $23.63 \%$ & & & & \\
\hline$B^{*} \quad \begin{array}{l}\text { Ilote: This is the } 2 \\
\text { EurosCoRE In }\end{array}$ & Calculate Cloge & & & & \\
\hline
\end{tabular}


6.4 Anhang 4: Fragebogen für telefonisches Follow-up

\section{Telefonbogen TAVI-Studie}

Name Patient:

Datum des Telefongesprächs:

Mortality:

Patient lebt

ㅁ Patient verstorben

Datum des Todes:

Sterbeort:

Todesursache:

Entlassungsbrief des letzten stationären Aufenthaltes:

Aktuelle Symptomatik:

- Tritt Luftnot auf?

- Bei welcher körperlichen Belastung tritt Luftnot auf? (Nach wie vielen Stufen Treppensteigen oder nach wie vielen Metern Gehen in der Ebene?)

- Treten Schmerzen in der Brust auf?

- Kam es seit der Operation zu einem Bewusstseinsverlust?

- Haben Sie insgesamt den Eindruck, dass sich seit der Operation ihre körperliche Leistungsfähigkeit verbessert hat?

- Würden Sie den Eingriff noch einmal durchführen lassen?

\section{Hospitalisationen nach TAVI:}

Waren Sie seit dem Eingriff an der Aortenklappe wieder im Krankenhaus?

Ist eines der folgenden Ereignisse eingetreten?

Schlaganfall $\square$

erneuter Eingriff an der Aortenklappe

Akute Herzschwāche (Wasser in der Lunge, Wasser in den Beinen, schwere Luftnot)

Stat. Anfenthalt 1: Datum

Krankenhaus

Ursache Entlassungsbrief

Stat. Anfenthalt 2: Datum Krankenhaus

Ursache

Entlassungsbrief

Stat. Aufenthalt 3: Datum Krankenhaus

Ursache Entlassungsbrief 


\section{Literaturverzeichnis}

Afilalo J, Eisenberg MJ, Morin JF, Bergman H, Monette J, Noiseux N, Perrault LP, Alexander KP, Langlois Y, Dendukuri N et al. (2010): Gait speed as an incremental predictor of mortality and major morbidity in elderly patients undergoing cardiac surgery. J Am Coll Cardiol $\underline{56}$, 1668-1676

Afilalo J, Mottillo S, Eisenberg MJ, Alexander KP, Noiseux N, Perrault LP, Morin JF, Langlois Y, Ohayon SM, Monette J et al. (2012): Addition of frailty and disability to cardiac surgery scores identifies elderly patients at high risk of mortality or major morbidity. Circ Cardiovasc Qual Outcomes $\underline{5}, 222-228$

Barili F, Pacini D, Capo A, Rasovic O, Grossi C, Alamanni F, Di BR, Parolari A (2013): Does EuroSCORE II perform better than its original versions? A multicentre validation study. Eur Heart J $\underline{34}, 22-29$

Ben-Dor I, Pichard AD, Gonzalez MA, Weissman G, Li Y, Goldstein SA, Okubagzi P, Syed Al, Maluenda G, Collins SD et al. (2010): Correlates and causes of death in patients with severe symptomatic aortic stenosis who are not eligible to participate in a clinical trial of transcatheter aortic valve implantation. Circulation $\underline{122}, 37-42$

Bonow RO, Carabello B, de Leon AC Jr et al. (1998): ACC/AHA guidelines for the management of patients with valvular heart disease: a report of the American College of Cardiology/American Heart Association Task Force on Practice Guidelines (Committee on Management of Patients With Valvular Heart Disease). J Am Coll Cardiol 32, 1486-1588

Boyd CM, Xue QL, Simpson CF, Guralnik JM, Fried LP (2005): Frailty, hospitalization, and progression of disability in a cohort of disabled older women. Am J Med 118, 1225-1231 
Buellesfeld L, Windecker S (2011): Transcatheter aortic valve implantation: the evidence is catching up with reality. Eur Heart J 32, 133-137

Carità P, Coppola G, Novo G, Caccamo G, Guglielmo M, Balasus F, Novo S, Castrovinci S, Moscarelli M, Fattouch K, Corrado E (2016): Aortic stenosis: insights on pathogenesis and clinical implications. J Geriatr Cardiol 13, 489-498

Chukwuemeka A, Borger MA, Ivanov J, Armstrong S, Feindel C, David T (2006): Valve surgery in octogenarians: a safe option with good medium-term results. J Heart Valve Dis $\underline{15}, 191-196$

Cribier, A, Eltchaninoff $\mathrm{H}$, Bash A, Borenstein N, Tron C, Bauer F, Derumeaux G, Anselme F, Laborde F, Leon MB (2002): Percutaneous transcatheter implantation of an aortic valve prosthesis for calcific aortic stenosis: first human case description. Circulation 106, 3006-3008

Figulla HR, Cremer J, Walther T, Gerckens U, Erbel R, Osterspey A, Zahn R (2009): Positionspapier zur kathetergeführten Aortenklappenimplantation. Kardiologe $\underline{3}, 199-206$

Fried LP, Tangen CM, Walston J, Newman AB, Hirsch C, Gottdiener J, Seeman T, Tracy R, Kop WJ, Burke G et al. (2001): Frailty in older adults: evidence for a phenotype. J Gerontol A Biol Sci Med Sci $\underline{56}$, M146-M156

Fried LP, Ferrucci L, Darer J, Williamson JD, Anderson G (2004): Untangling the concepts of disability, frailty and comorbidity: implications for improved targeting and care. J Gerontol A Biol Sci Med Sci $\underline{59}$, 255-263

Green P, Woglom AE, Genereux P, Maurer MS, Kirtane AJ, Hawkey M, Schnell S, Sohn J, Moses JW, Leon MB et al. (2012 a): Gait speed and 
dependence in activities of daily living in older adults with severe aortic stenosis. Clin Cardiol $\underline{35}$, 307-314

Green P, Woglom AE, Genereux P, Daneault B, Paradis JM, Schnell S, Hawkey M, Maurer MS, Kirtane AJ, Kodali S et al. (2012 b): The impact of frailty status on survival after transcatheter aortic valve replacement in older adults with severe aortic stenosis: a single center experience. JACC Cardiovasc Interv $\underline{9}, 974-981$

Grube E, Laborde JC, Gerckens U, Felderhoff T, Sauren B, Buellesfeld L, Mueller R, Menichelli M, Schmidt T, Zickmann B, et al. (2006): Percutaneous implantation of the CoreValve self-expanding valve prosthesis in high-risk patients with aortic valve disease: the Siegburg first-in-man study. Circulation 114, 1616-1624

Holmes DR, Mack MJ (2015): Uncertainty and Possible Subclinical Valve Leaflet Thrombosis. N Engl J Med $\underline{373}$, 2080-2082

Horstkotte D, Loogen F (1988): The natural history of aortic valve stenosis Eur Heart J $\underline{9}, 57-64$

Inouye SK, Peduzzi PN, Robison JT, Hughes JS, Horwitz RI, Concato J (1998): Importance of functional measures in predicting mortality among older hospitalized patients. JAMA $\underline{15}, 1187-1193$

lung B, Baron G, Butchart EG, Delahaye F, Gohlke-Barwolf C, Levang OW, Tornos P, Vanoverschelde JL, Vermeer F, Boersma E et al. (2003): A prospective survey of patients with valvular heart disease in Europe: The Euro Heart Survey on Valvular Heart Disease. Eur Heart J 24, 1231-1243

lung B, Cachier A, Baron G, Messika-Zeitoun D, Delahaye F, Tornos P, Gohlke-Barwolf C, Boersma E, Ravaud P, Vahanian A (2005): Decision- 
making in elderly patients with severe aortic stenosis: Why are so many denied surgery? Eur Heart J $\underline{26}, 1231-1243$

Kaplan EL, Meier P (1958): Non parametric estimation from incomplete observations. J Am Stat Ass $\underline{53}, 457$

Kappetein AP, Head SJ, Genereux P, Piazza N, Van Mieghem NM, Blackstone EH, Brott TG, Cohen DJ, Cutlip DE, van Es GA et al. (2012): Updated standardized endpoint definitions for transcatheter aortic valve implantation: the Valve Academic Research Consortium-2 consensus document. Eurolntervention $\underline{8}, 782-795$

Katz S, Ford AB, Moskowitz RW, Jackson BA, Jaffe MW (1963): Studies of illness in the aged. The Index of ADL: A standardized measure of biological and psychosocial function. JAMA $\underline{185}, 914-919$

Kodali SK, Williams MR, Smith CR, Svensson LG, Webb JG, Makkar RR, Fontana GP, Dewey TM, Thourani VH, Pichard AD et al. (2012): Two-year outcomes after transcatheter or surgical aortic-valve replacement. N Engl J Med $\underline{366}, 1686-1695$

Lang RM, Bierig M, Devereux RB, Flachskampf FA, Foster E, Pellikka PA, Picard MH, Roman MJ, Seward J, Shanewise JS et al. (2005): Recommendations for chamber quantification: a report from the American Society of Echocardiography's Guidelines and Standards Committee and the Chamber Quantification Writing Group, developed in conjunction with the European Association of Echocardiography, a branch of the European Society of Cardiology. J Am Soc Echocardiogr 18, 1440-1463

Lee DH, Buth KJ, Martin BJ, Yip AM, Hirsch GM (2010): Frail patients are at increased risk for mortality and prolonged institutional care after cardiac surgery. Circulation $\underline{121}, 973-978$ 
Leon MB, Smith CR, Mack M, Miller DC, Moses JW, Svensson LG, Tuzcu EM, Webb JG, Fontana GP, Makkar RR et al. (2010): Transcatheter aortic-valve implantation for aortic stenosis in patients who cannot undergo surgery. N Engl J Med $\underline{363}, 1597-1607$

Leon MB, Piazza N, Nikolsky E, Blackstone EH, Cutlip DE, Kappetein AP, Krucoff MW, Mack M, Mehran R, Miller C et al. (2011): Standardized endpoint definitions for transcatheter aortic valve implantation clinical trials: a consensus report from the Valve Academic Research Consortium. Eur Heart J $\underline{32}, 205-217$

Lichtenstein SV, Cheung A, Ye J, Thompson CR, Carere RG, Pasupati S, Webb JG (2006): Transapical transcatheter aortic valve implantation in humans: initial clinical experience. Circulation 114, 591-596

Makkar RR, Fontana GP, Jilaihawi H, Kapadia S, Pichard AD, Douglas PS, Thourani VH, Babaliaros VC, Webb JG, Herrmann HC et al. (2012): Transcatheter aortic-valve replacement for inoperable severe aortic stenosis. N Engl J Med 366, 1696-1704

Mok M, Nombela-Franco L, Urena M, DeLarochelliere R, Doyle D, Ribeiro HB, Cote M, Pibarot P, DeLarochelliere H, Laflamme L et al. (2013): Prognostic value of exercise capacity as evaluated by the 6 -minute walk test in patients undergoing transcatheter aortic valve implantation. J Am Coll Cardiol $\underline{61}, 897-898$

Nashef SA, Roques F, Michel P, Gauducheau E, Lemeshow S, Salamon R (1999): European system for cardiac operative risk evaluation (EuroSCORE). Eur J Cardiothorac Surg 16, 9-13 
Nkomo VT, Gardin JM, Skelton TN, Gottdiener JS, Scott CG, EnriquezSarano M (2006): Burden of valvular heart diseases: a population-based study. Lancet $\underline{368}, 1005-1011$

Otto C (2008): Calcific Aortic Stenosis - Time to look more closely at the Valve. N Engl J Med 359, 1395-1398

Otto CM, Mickel MC, Kennedy JW, Alderman EL, Bashore TM, Block PC, Brinker JA, Diver D, Ferguson J, Holmes DR Jr et al. (1994): Three-year outcome after balloon aortic valvuloplasty. Insights into prognosis of valvular aortic stenosis. Circulation $\underline{89}, 642-650$

Rockwood K (2005): Frailty and its definition: a worthy challenge. J Am Geriatr Soc 53, 1069-1070

Rodes-Cabau J, Webb JG, Cheung A, Ye J, Dumont E, Osten M, Feindel CM, Natarajan MK, Velianou JL, Martucci G, DeVarennes B et al. (2012): Long-term outcomes after transcatheter aortic valve implantation: insights on prognostic factors and valve durability from the Canadian multicenter experience. J Am Coll Cardiol $\underline{60}, 1864-1875$

Roques F, Michel P, Goldstone AR, Nashef SA (2003): The logistic EuroSCORE. Eur Heart J $\underline{24}$, 881-882

Rosenhek R, lung B, Tornos $\mathrm{P}$, Antunes MJ, Prendergast BD, Otto CM, Kappetein AP, Stepinska J, Kaden JJ, Naber CK et al. (2012): ESC Working Group on Valvular Heart Disease Position Paper: assessing the risk of interventions in patients with valvular heart disease. Eur Heart J $\underline{33}$, 822-8, 828a, 828b

Schaefer A, Bertram H (2010): Transcatheter valve interventions for heart valve diseases. Internist (Berl). $\underline{51}, 1480-1491$ 
Smith CR, Leon MB, Mack MJ, Miller DC, Moses JW, Svensson LG, Tuzcu EM, Webb JG, Fontana GP, Makkar RR et al. (2011): Transcatheter versus surgical aortic-valve replacement in high-risk patients. $\mathrm{N}$ Engl J Med $\underline{364}, 2187-2198$

Stortecky S, Schoenenberger AW, Moser A, Kalesan B, Juni P, Carrel T, Bischoff S, Schoenenberger CM, Stuck AE, Windecker S et al. (2012): Evaluation of multidimensional geriatric assessment as a predictor of mortality and cardiovascular events after transcatheter aortic valve implantation. JACC Cardiovasc Interv $\underline{5}$, 489-496

Vahanian A, Alfieri O, Al-Attar N, Antunes M, Bax J, Cormier B, Cribier A, De JP, Fournial G, Kappetein AP et al. (2008): Transcatheter valve implantation for patients with aortic stenosis: a position statement from the European Association of Cardio-Thoracic Surgery (EACTS) and the European Society of Cardiology (ESC), in collaboration with the European Association of Percutaneous Cardiovascular Interventions (EAPCI). Eur Heart J $\underline{29}, 1463-1470$

Vahanian A, Alfieri O, Andreotti F, Antunes MJ, Baron-Esquivias G, Baumgartner H, Borger MA, Carrel TP, De BM, Evangelista A et al. (2012): Guidelines on the management of valvular heart disease. Eur Heart $\mathrm{J} \underline{33}$, 2451-2496

Webb JG, Chandavimol M, Thompson CR, Ricci DR, Carere RG, Munt BI, Buller CE, Pasupati S, Lichtenstein S (2006): Percutaneous aortic valve implantation retrograde from the femoral artery. Circulation $\underline{113}, 842-50$

Zoghbi WA, Enriquez-Sarano M, Foster E, Grayburn PA, Kraft CD, Levine RA, Nihoyannopoulos P, Otto CM, Quinones MA, Rakowski H et al. (2003): Recommendations for evaluation of the severity of native valvular regurgitation with two-dimensional and Doppler echocardiography. J Am Soc Echocardiogr 16, 777-802 


\section{Danksagung}

Herrn Prof. Dr. Gerd Hasenfuß, Direktor der Klinik für Kardiologie und Pneumologie am Universitätsklinikum Göttingen, bin ich sehr dankbar für die Möglichkeit, die vorliegende Promotionsschrift in seiner Abteilung erstellen zu dürfen.

Bei Herrn Prof. Dr. Wolfgang Schillinger möchte ich mich ganz herzlich für die Aufnahme in seine Arbeitsgruppe und die Überlassung dieses interessanten Dissertationsthemas bedanken. Jederzeit konnte ich mich auf seine hilfreiche und freundliche Unterstützung verlassen.

Mein ganz besonderer Dank gilt Frau Dr. Miriam Puls. Ihre intensive und freundschaftliche Betreuung und ihre außergewöhnliche Unterstützung haben wesentlich zum Gelingen dieser Doktorarbeit beigetragen.

Bedanken möchte ich mich weiterhin bei Frau Dr. Annalen Bleckmann für das große Engagement bei der Auswertung der statistischen Ergebnisse.

Ebenfalls bedanke ich mich bei Frau Mühlhausen, Frau Quast-Borgelt und den Mitarbeitern der Leitstelle Innere Medizin für ihre Geduld und Hilfe bei der Organisation der Patientenakten. Nicht zu vergessen, möchte ich auch Frau Tanja Viel für die Einführung in sämtliche Abläufe der Aufgaben als studentische Hilfskraft danken. 